\title{
Simulating the Aerodynamic Performance and Wake Dynamics of a Vertical-Axis Wind Turbine
}

\author{
Frank Scheurich* Timothy M. Fletcher ${ }^{\dagger} \quad$ Richard E. Brown ${ }^{\ddagger}$ \\ Rotor Aeromechanics Laboratory \\ Department of Aerospace Engineering \\ University of Glasgow \\ Glasgow, G12 8QQ, United Kingdom
}

\begin{abstract}
The accurate prediction of the aerodynamics and performance of verticalaxis wind turbines is essential if their design is to be improved but poses a significant challenge to numerical simulation tools. The cyclic motion of the blades induces large variations in the angle of attack of the blades that can manifest as dynamic stall. In addition, predicting the interaction between the blades and the wake developed by the rotor requires a high-fidelity representation of the vortical structures within the flow field in which the turbine operates. The aerodynamic performance and wake dynamics of a Darrieustype vertical-axis wind turbine consisting of two straight blades is simulated using Brown's Vorticity Transport Model. The predicted variation with azimuth of the normal and tangential force on the turbine blades compares well with experimental measurements. The interaction between the blades and the vortices that are shed and trailed in previous revolutions of the turbine is shown to have a significant effect on the distribution of aerodynamic loading on the blades. Furthermore, it is suggested that the disagreement between experimental and numerical data that has been presented in previous studies arises because the blade-vortex interactions on the rotor were not modelled with sufficient fidelity.
\end{abstract}

\footnotetext{
* Postgraduate Research Student, f.scheurich@aero.gla.ac.uk (corresponding author)

Post-doctoral Research Assistant, t.fletcher@eng.gla.ac.uk

$\ddagger$ Mechan Chair of Engineering, r.brown@aero.gla.ac.uk
} 


\section{Nomenclature}

$A R \quad$ aspect ratio, $b / c$

$b \quad$ blade span

$c \quad$ local chord length of the blade section

$C_{d} \quad$ sectional drag coefficient, $D^{\prime} / \frac{1}{2} \rho c u_{b}^{2}$

$C_{l} \quad$ sectional lift coefficient, $L^{\prime} / \frac{1}{2} \rho c u_{b}^{2}$

$C_{n} \quad$ normal force coefficient, $F_{n} / \frac{1}{2} \rho c u_{b}^{2}$

$C_{t} \quad$ tangential force coefficient, $F_{t} / \frac{1}{2} \rho c u_{b}^{2}$

$D^{\prime} \quad$ sectional drag

$F_{n} \quad$ sectional force acting normal to the chord

$F_{N} \quad$ force acting normal to the blade chord

$F_{n}^{*} \quad$ sectional non-dimensional normal force, $F_{n} / \frac{1}{2} \rho c V_{\infty}^{2}$

$F_{N}^{*} \quad$ non-dimensional normal force, $F_{N} / \frac{1}{2} \rho b c V_{\infty}^{2}$

$F_{t} \quad$ sectional force acting tangential to the chord

$F_{T} \quad$ force acting tangential to the blade chord

$F_{t}^{*} \quad$ sectional non-dimensional tangential force, $F_{t} / \frac{1}{2} \rho c V_{\infty}^{2}$

$F_{T}^{*} \quad$ non-dimensional tangential force, $F_{T} / \frac{1}{2} \rho b c V_{\infty}^{2}$

$L^{\prime} \quad$ sectional lift

$R \quad$ rotor radius

Re average blade Reynolds number, $\Omega R c / \nu$

$S \quad$ vorticity source term

$u \quad$ flow velocity

$u_{b} \quad$ flow velocity relative to the blade

$V_{\infty} \quad$ free stream velocity

$\alpha \quad$ angle of attack

$\lambda \quad$ tip speed ratio, $\Omega R / V_{\infty}$

$\nu \quad$ kinematic viscosity

$\psi \quad$ azimuth angle

$\rho$ density

$\omega \quad$ vorticity

$\omega_{b} \quad$ bound vorticity

$\Omega \quad$ angular velocity of the rotor 


\section{Introduction}

The number of wind turbines that are being constructed is increasing substantially. This is mainly in response to the perceived need to reduce emissions of carbon and other pollutants. The current generation of wind turbines that are being deployed around the world feature, almost exclusively, a three-bladed rotor with a horizontal-axis configuration. In recent years, however, there has been a resurgence of interest in both large-scale and small-scale verticalaxis wind turbines. The development of large-scale vertical-axis wind turbines is mainly a response to a plateau in the improvement of the aerodynamic performance of horizontal-axis wind turbines. The research in small-scale vertical-axis wind turbines, with rotor diameters of only several meters, is motivated by the future demand for a decentralised and sustainable energy supply in cities and rural communities.

Vertical-axis wind turbines have a drive train that is situated on the ground, thereby reducing the loads on the tower, and facilitating the maintenance of its systems. In addition, vertical-axis wind turbines are, by design, insensitive to the direction of the wind, and therefore do not require a yaw control system. The principal advantage of these features is to enable a design that alleviates the material stress on the tower and requires fewer mechanical components.

A vertical-axis wind turbine is called a Darrieus-type turbine when it is driven by aerodynamic lift. The concept was patented in the United States by Georges Darrieus in 1931 [1]. The aerodynamic simulation of Darrieus-type vertical-axis wind turbines poses a significant challenge. This is because the angle of attack of its blades varies cyclically with rotor azimuth during its operation. Dynamic stall can thus occur, particularly at low tip speed ratios, when the amplitude of the oscillation of the angle of attack becomes large enough to induce flow separation.

At high tip speed ratios, the amplitude of the oscillations in the angle of attack of the blades is significantly smaller than at low tip speed ratios. Indeed, the blades then operate predominantly within the regime in which the lift coefficient varies linearly with incidence. The interaction between each of the turbine blades and either their own wakes, or the wakes developed downwind of the other blades, influences the aerodynamic loading on the blades more greatly when the turbine is operating at high tip speed ratios, however. This is because at high tip speed ratios the wake developed by each of the turbine blades convects downstream relatively slowly when compared to the rotational speed of the turbine, allowing each blade to encounter its own wake in the subsequent revolution. According to Klimas [2], the interaction between the blades and the wake of the turbine is considered to be one of the most critical problems in the numerical modelling of the aerodynamics of vertical-axis wind turbines. Indeed, the interactions between the blades of the turbine and the vortices in its wake produce impulsive variations in the aerodynamic loading on the blades of the turbine, but these interactions are notoriously difficult to simulate accurately.

Historically, a range of theoretical and computational aerodynamic methods has been used to model the flow environment around vertical-axis wind turbines. These include streamtube models [3,4], vortex lattice models [5], and so-called cascade models [6]. In recent years, however, improvements in the availability of high-performance computing have allowed the aerodynamics of wind turbines to be computed from first principles using the Navier-Stokes equations. Hansen and Sørensen [7], as well as Simão Ferreira et al. [8], have used computational schemes that solve the Reynolds-Averaged Navier-Stokes (RANS) equations to simulate 
the two-dimensional aerodynamics of an aerofoil while in a planar, cyclic motion designed to emulate that of the blades of a vertical-axis wind turbine, whilst detached-eddy simulations of a similar aerofoil configuration have been performed by Horiuchi et al. [9].

The case described in the present paper comprises a Darrieus-type vertical-axis wind turbine consisting of two straight blades operating at a tip speed ratio of five. This turbine was studied experimentally by Strickland et al. [10] who measured the variation of the blade loads with azimuth. Strickland et al. [10] also carried out complementary numerical studies using a vortex lattice method. Their predicted results agreed reasonably well with their experimental data. Improved results were achieved by Ponta and Jacovkis [11] using a computational model which coupled the far field, where a free vortex scheme was employed, with the near aerofoil region, where a finite element analysis was used to calculate the aerodynamic forces by integrating the fluid momentum equation. Both numerical studies, however, yielded inaccurate predictions of the blade loads immediately downwind of the turbine. As will be shown in the present paper, this is where the strongest interference occurs between the blade and the vortices within the wake system that is produced by the turbine rotor.

In this paper, Brown's Vorticity Transport Model [12] has been used to predict the normal and tangential forces acting on the blades of a straight-bladed Darrieus turbine that has been configured to be geometrically analogous to the experimental device used by Strickland et al. [10]. It is shown that the interaction between the blades and the vortices that are shed and trailed in previous revolutions has a significant effect on the distribution of aerodynamic loading on the blade. Furthermore, it is suggested that the disagreement between experimental and numerical data that has been presented in previous studies that were carried out by Strickland et al. [10] and Ponta and Jacovkis [11], was most likely a result of the interactions between the blades of the turbine and the wake that is developed by the turbine having not been modelled with sufficient fidelity.

\section{Computational Aerodynamics}

The aerodynamic performance and wake dynamics of a vertical-axis wind turbine have been simulated using the Vorticity Transport Model (VTM) developed by Brown [12], and extended by Brown and Line [13]. The VTM enables the simulation of wind turbine aerodynamics and performance by providing an accurate representation of the dynamics of the wake that is generated by the turbine rotor. The time-dependent Navier-Stokes equations are discretised in three-dimensional finite-volume form using a structured Cartesian mesh within the fluid domain surrounding the turbine rotor. Using the assumption that the wake is incompressible, the Navier-Stokes equations are cast into the vorticity-velocity form

$$
\frac{\partial}{\partial t} \omega+u \cdot \nabla \omega-\omega \cdot \nabla u=S+\nu \nabla^{2} \omega
$$

allowing the temporal evolution of vorticity distribution $\omega=\nabla \times u$ in the flow surrounding the rotor to be modelled. The advection, stretching, and diffusion terms within the vorticity transport equation describe the changes in the vorticity field, $\omega$, with time at any point in space, as a function of the velocity field, $u$, and the viscosity, $\nu$. The vorticity source term, $S$, is used to account for the creation of vorticity at solid surfaces immersed within the fluid. The vorticity source term is determined as the sum of the temporal and spatial variations in 
the bound vorticity, $\omega_{b}$, on the turbine blades so that

$$
S=-\frac{d}{d t} \omega_{b}+u_{b} \nabla \cdot \omega_{b}
$$

The first term in Equation 2 represents the shed vorticity and the second term represents the trailed vorticity from the blade. The bound vorticity distribution on the blades of the rotor is modelled using an extension of lifting-line theory. The lifting-line approach has been appropriately modified by the use of two-dimensional experimental data in order to represent the real performance of any given airfoil. The turbine that has been investigated operates at a tip speed ratio of five in order to compare the simulation results to experimental measurements made by Strickland et al. [10]. At this tip speed ratio, the effects of dynamic stall have historically been considered to be small. Indeed, Laneville and Vittecoq [14] showed, in an experiment with a straight-bladed Darrieus turbine using blades with a NACA 0018 aerofoil section and operating at an average Reynolds number of 38000, that dynamic stall is minimal or absent when the tip speed ratio is greater than four. For this reason no representation of dynamic stall was included within the VTM model for the blade aerodynamic loading in the initial simulations presented in this paper. Some of the results presented later in this paper suggest, however, that dynamic stall, induced by the interaction of the blades and the wake of the turbine, may indeed occur near to the tip of the blades even during operation at a tip speed ratio of five. Therefore, additional simulations have been carried out using the VTM model in conjunction with a dynamic stall model that is based on the semi-empirical model that has been suggested by Leishman and Beddoes [15]. The Leishman-Beddoes model was originally developed to simulate the effect of dynamic stall on the blades of a helicopter. Gupta and Leishman [16] demonstrated, however, that a modified version of the original LeishmanBeddoes model can be used to represent the dynamic stall of airfoils of the type that are typically used for horizontal-axis wind turbines. The Leishman-Beddoes-type dynamic stall model that is implemented in the VTM is based on the third generation dynamic stall model as proposed by Beddoes [17], but has been modified, as suggested by Niven and Galbraith [19] and Sheng, Galbraith and Coton [18] to account properly for vortex inception at low Mach numbers, and negative tangential force coefficients in deep dynamic stall, respectively.

The velocity field is related to the vorticity field by using a Cartesian fast multipole method to invert the differential form of the Biot-Savart law

$$
\nabla^{2} u=-\nabla \times \omega
$$

The fast multipole method is used, in conjunction with an adaptive grid in which cells are only present within the calculation when the vorticity within them is non-zero, to dramatically reduce the computational cost of each simulation when compared to an equivalent calculation performed on a fixed grid. As the vorticity moves to a new location, new cells are created and any cells that no longer contain vorticity are destroyed. Thus, the grid structure is free to follow the evolution of the wake, eliminating the requirement for explicit numerical boundary conditions at the edge of the computational domain and increasing the computational efficiency of the method. An assumption is made that the Reynolds number within the computational domain is sufficiently high so that the equations governing the flow in the wake of the rotor may be solved in inviscid form. The numerical diffusion of vorticity within the flow field surrounding the wind turbine is kept at a very low level by using a Riemann problem technique based on the Weighted Average Flux method developed by Toro [20] to advance Equation 1 through 
time. This approach permits many rotor revolutions to be captured without significant spatial smearing of the wake structure, in contrast to the performance of more conventional CFD techniques based on the pressure-velocity formulation of the Navier-Stokes equations. The wake does naturally dissipate with time, however, because of the inherent instability of the vortical structures of which it is composed.

Fletcher et al. [21] validated VTM predictions of the performance of a horizontal-axis wind turbine against experimental data that was published by Hand et al. [22], and compared VTM predictions of wind turbine performance to those of more conventional RANS methods. Fletcher et al. [23] have also used the VTM successfully to investigate the aerodynamic interaction between horizontal-axis wind turbines within a wind farm.

\section{Turbine Model}

The properties of the wind turbine that has been investigated are summarised in Table 1 . The VTM allows grid levels of different cell size to be specified. Coarser grids are used with increasing distance from the turbine centre in order to reduce the computational costs of the simulations and to focus resources closer to where the wake needs to be most highly resolved. Figure 1 illustrates the configuration of the grid after seven rotor revolutions have elapsed. At this point in the computation, the wake is contained within a series of grids with four different levels of refinement. In the numerical study which is presented in this paper, the finest computational grid, containing cells with an edge length of $R / 44$, extends to four rotor radii downstream from the turbine centre.

For the simulation of the blade aerodynamics, the surface of the blade was discretised into 32 panels. Panels were concentrated at the blade tips in order to resolve accurately the formation of the tip vortices. The orientation of the blades and specification of the azimuth angle of the turbine with respect to the reference blade ('blade 1') is shown in Figure 2.

The predicted performance of the turbine was compared with data that was obtained from experiments carried out in water in a tow tank. The experimental data was originally published by Strickland et al. [10]. In the experiment, strain gauges were used to record the variation with azimuth of the normal and tangential forces on the blades of the turbine. As shown in Figure 3(a), one end of the blade pierced the water surface while the other end of the blade operated close to the bottom of the tow tank. The gap between the blade tip and the bottom of the tank was stated as being less than $15 \mathrm{~cm}$. In their analysis, however, Strickland et al. [10] compared their experimental data with numerical results for the non-dimensional tangential and normal forces for a two-dimensional rotor under the assumption that the presence of the tank bottom and the free surface would reduce three-dimensional effects. This assumption is open to some doubt given the configuration of the turbine within the tow tank, however. As shown in the present paper, the gap between the blade and the bottom of the tow tank leads to the formation of tip vortices which convect downstream and interact with the blades of the turbine. These blade-vortex interactions are simply not present in a strictly two-dimensional flow field.

In the numerical study presented in this paper, the bottom of the tow tank is represented by a ground plane. This plane was set at a distance of $10 \mathrm{~cm}$ from the blade tip in order to simulate the gap between the bottom of the tow tank and the end of the blade, as depicted in Figure 3(b). In the experiment, the aspect ratio of the submerged portion of the blade was five. In the numerical study, the water surface was not modelled, however. Instead, the 
blade span was increased to yield an aspect ratio of 15 in order to prevent any interaction between the lower portion of the blade and the vortices trailed from the upper tip of the blade. Consequently, when comparing the predicted forces with the experimental measurements, the VTM-calculated aerodynamic forces acting only on the lower portion of the blade, in other words, only on that part of the blade which is equivalent to the submerged portion of the blade in the experiment, has been presented in order to provide a more realistic comparison of the two systems.

\section{Aerofoil Data}

The lifting-line method that is used within the VTM to model the distribution of the bound vorticity on the blades of the turbine relies upon the specification of the aerofoil lift and drag coefficients as functions of angle of attack. Vertical-axis wind turbines can operate at relatively low Reynolds numbers with large cyclic variations in the angle of attack at the blades. Unfortunately, aerofoil performance data for such operating conditions is scarce within the published literature. In addition, Tangler [24] demonstrated that measurements of the performance of aerofoils that are made in different wind tunnels can vary significantly.

Simulations based on two different aerofoil data sets were carried out in order to allow the effect on the numerical results of variability in the aerofoil performance to be quantified. Experimental data for the variation of the lift and drag coefficients with angle of attack, as measured by Althaus [25], are compared with data computed using XFoil [26] in Figures 4 and 5, respectively. Both the measured and predicted lift and drag coefficients were obtained for a Reynolds number of 40000 . The computed stall angle is $8^{\circ}$, whereas the experimental measurements indicate that stall occurs at an angle of attack that is slightly greater than $8^{\circ}$. Both the computed and experimental data for the lift and drag coefficient agree well for angles of attack between $0^{\circ}$ and $5^{\circ}$ as well as for angles of attack greater than $9^{\circ}$, however. For angles of attack between $5^{\circ}$ and $9^{\circ}$, the experimental measurements suggest a lower lift coefficient and a slightly higher drag coefficient when compared to the computed data.

Given the definition of the azimuthal orientation of the blades shown in Figure 2, the maximum and minimum velocity, and consequently the maximum and minimum local Reynolds number, are experienced by the blades when oriented at azimuth angles of $0^{\circ}$ and $180^{\circ}$, respectively. At azimuth angles of $0^{\circ}$ and $180^{\circ}$, the angle of attack of the blades is very low. The effect of the Reynolds number on the variation of lift coefficient with angle of attack at small angles is known to be marginal, however, as demonstrated by Abbott and von Doenhoff [27], amongst others. Indeed, the primary influence of the Reynolds number on the variation of lift coefficient with angle of attack is in the stall region. This observation justifies the use, in all of the simulations presented in this paper, of aerofoil data based on the average Reynolds number of the rotor blades. 


\section{Blade Aerodynamic Loading}

By adopting the notation that was used by Strickland et al. [10], the normal and tangential forces that act on a blade section can be written in terms of the sectional non-dimensional normal force $F_{n}^{*}$ and sectional non-dimensional tangential force $F_{t}^{*}$. The sectional non-dimensional forces are related to the sectional normal and tangential force coefficients $C_{n}$ and $C_{t}$ by

$$
\begin{aligned}
& F_{n}^{*}=\frac{F_{n}}{1 / 2 \rho c V_{\infty}^{2}}=C_{n}\left(\frac{u_{b}}{V_{\infty}}\right)^{2} \\
& F_{t}^{*}=\frac{F_{t}}{1 / 2 \rho c V_{\infty}^{2}}=C_{t}\left(\frac{u_{b}}{V_{\infty}}\right)^{2}
\end{aligned}
$$

where $F_{n}$ and $F_{t}$ are the sectional normal and tangential forces, $V_{\infty}$ is the free stream velocity, and $u_{b}$ is the local velocity at the blade section. This velocity $u_{b}$ comprises contributions from the circumferential velocity of the blade, the free stream velocity and the velocity component that is induced by the wake. The coefficients $C_{n}$ and $C_{t}$ are related to the aerofoil lift and drag coefficients $C_{l}$ and $C_{d}$ by

$$
\begin{aligned}
& C_{n}=-C_{l} \cos \alpha-C_{d} \sin \alpha \\
& C_{t}=C_{l} \sin \alpha-C_{d} \cos \alpha
\end{aligned}
$$

where $\alpha$ is the local angle of attack. Finally, the non-dimensional forces acting on the entire lower portion of the blade, in other words the portion that is equivalent to the submerged portion of the blade in the experiment, are denoted as $F_{N}$ and $F_{T}$.

The local blade velocity varies cyclically with azimuth due to the cyclic variation in the direction of the incident flow at the blade. Indeed, the geometric angle of attack of the blade, $\alpha$, is a function only of the tip speed ratio, $\lambda$, and the azimuth angle, $\psi$, such that

$$
\alpha=\arctan \left(\frac{\sin \psi}{\lambda+\cos \psi}\right) \text {. }
$$

Figure 6 shows the variation with azimuth of the geometric angle of attack of the blade (crosses). Given the symmetry about $\alpha=0^{\circ}$ of the variation of the geometric angle of attack of the blade, an over-simplistic analysis of the aerodynamics of the system may lead one to the conclusion that the variation in loading on the blades with azimuth should also possess equivalent inherent symmetry, centred on an azimuth of $0^{\circ}$. That this is not the case is revealed in Figure 7, which shows the VTM-predicted variation of the non-dimensional normal force, $F_{n}^{*}$, and the non-dimensional tangential force, $F_{t}^{*}$, with azimuth angle $\psi$ for the section at the mid-span of the blade. The reason for this seeming anomaly, even in the case of a blade with such high aspect ratio, is revealed in Figure 6 where the aerodynamic angle of attack of the centre section of the blade is also plotted as a function of blade azimuth (solid line). As the blade passes downstream of the axis of rotation, the flow that is induced by the wake of the turbine, and particularly in this case the effect of the shed vorticity from the preceding blades, reduces the aerodynamic angle of attack of this section of the blade significantly below the geometric angle of attack. This results in a marked azimuthal asymmetry in the loading that is produced at the mid-span of the blade. 
Importantly though, Figure 6 shows the computed aerodynamic angle of attack at the midspan of the blade to only marginally exceed the static stall angle of the aerofoil, and then only when the blade is directly upstream of the axis of rotation of the turbine. Thus, in agreement with previous studies by Laneville and Vittecoq [14], it can be inferred that dynamic stall is absent for the particular tip speed ratio at which the turbine was operated - at least at the mid-span of the blade.

The flow at the mid-span is least affected by three-dimensional aerodynamic effects, and thus the behaviour of the aerodynamic loading at this blade station provides the closest approximation to the two-dimensional aerodynamic performance of the blade aerofoil. Previous computations of the two-dimensional aerodynamics of the aerofoil in a planar, cyclic motion designed to emulate that of the mid-section of the blade, have been conducted by Strickland et al. [10] and by Ponta and Jacovkis [11], and comparison with this data provides the opportunity to verify, in part, the predictions of the VTM. In Figure 7, the VTM-predicted variation of the non-dimensional normal force, $F_{n}^{*}$, and the non-dimensional tangential force, $F_{t}^{*}$, with azimuth angle, $\psi$, for the section at mid-span of the blade (using both sets of aerofoil data described earlier) is compared to similar data obtained during these previous simulations. Generally, the VTM-predicted forces for the section at the mid-span of the blade are in very good agreement with the earlier numerical predictions - particularly with those obtained by Ponta and Jacovkis [11] - lending significant confidence in the predictive capabilities of the VTM. The differences in the VTM-simulated forces that are obtained when using the XFoil aerofoil data [26] and when using Althaus's experimental data [25] can be related directly to the differences between the two sets of aerofoil data. Indeed, the comparison allows some appreciation to be gained of the effect of the choice of two-dimensional aerofoil data on the predicted performance of this type of system. For instance, Figure 6 shows the angle of attack of the blade to be approximately $8^{\circ}$ near the maximum in the blade loading that is located close to $90^{\circ}$ azimuth. The relative under-prediction of the maximum loading on the blade when using Althaus's data is entirely consistent with Figures 4 and 5 which show the lift coefficients predicted by XFoil to be approximately $15 \%$ higher, for angles of attack between $5^{\circ}$ and $8^{\circ}$, than suggested by Althaus's data set.

Further confirmation of the predictions of the VTM may be obtained by comparing the integrated load on the lower part of the simulated blade to the loads on the submerged portion of the blade that were measured in the experiment conducted by Strickland et al. [10]. This comparison is presented in Figure $8(\mathrm{a})$ for the non-dimensional normal force, $F_{N}^{*}$, and Figure 8(b) for the non-dimensional tangential force, $F_{T}^{*}$. The figure shows very good overall agreement between the experimental measurements and the predicted forces on the submerged portion of the blade, with the exception of within a small range of azimuth angles between $225^{\circ}$ and $315^{\circ}$, in other words as the blade passes directly downstream of the axis of rotation of the turbine. Over this range of azimuth angles, there is a marked under-prediction of the normal force that is produced by the turbine and, similarly, a very obvious over-prediction of the tangential force.

In contrast to the situation at the mid-span of the blade, however, the flow near the tip of the blade cannot be assumed to be two-dimensional. From a modelling perspective, an appreciation of the magnitude and extent of the additional three-dimensional aerodynamic effects that may need to be accounted for, for instance when correcting the predictions of simpler two-dimensional models for the performance of such devices, would be very useful. Figure 9 compares the variation with azimuth of the aerodynamic angle of attack at the mid- 
span of the blade to that at several spanwise locations along the lower portion of the blade near to the blade tip (see Figure 13 where these locations are illustrated). A series of sharp, localised perturbations to the aerodynamic angle of attack of the blade are seen to extend a considerable distance inboard from the tip of the blade. The VTM predictions of the associated variation of the blade loading with azimuth, at the same spanwise stations, are compared in Figure 10, and exhibit corresponding features close to an azimuth of $270^{\circ}$ where the blade is directly downstream of the axis of rotation of the turbine.

Figures 11 and 12 show the variation of spanwise distribution of blade loading over one complete revolution of the turbine. The distribution of the loading along the blade in the upstream part of the revolution, between $0^{\circ}$ and $180^{\circ}$ azimuth, differs significantly to that in the downstream part of the revolution, between $180^{\circ}$ and $360^{\circ}$. In the upstream part of the revolution the blade loading is characterized by a smooth distribution of the aerodynamic forces which decreases steadily in magnitude towards the tip of the blades. The distribution of the loading along the blade in the downstream part of the revolution, in comparison, is characterized by series of impulsive and abrupt localised perturbations with a far more complex structure.

The localised perturbations to the angle of attack of the blade and the blade loading appear to be caused directly by aerodynamic interaction with the vorticity that is trailed from the lower tips of the blades of the turbine during previous revolutions. These blade-vortex interactions are visualised in Figure 13 by showing the vorticity distribution that is produced by the rotor on a plane oriented perpendicular to the ground, so that it contains the axis of rotation of the turbine and is aligned with the free stream velocity vector, at the instant of time when the reference blade ('blade $1^{\prime}$ ') is located at $270^{\circ}$ azimuth. The flow field is represented using contours of the component of vorticity perpendicular to the plane. The dark rendering corresponds to vorticity with a clockwise sense, and the light rendering to vorticity with an anticlockwise sense.

Figure 13 shows a concentrated stream of vorticity to interact very closely with the reference blade ('blade 1') as it passes downstream of the axis of rotation of the turbine. The location of this interaction, at a spanwise location of approximately $z / b=0.5$ on the lower portion of the blade, is entirely consistent with the variation of aerodynamic angle of attack along the span of the blade that is shown in Figure 9.

Most importantly, though, Figure 9 shows the aerodynamic angle of attack of the sections near the lower tip of the blade near to $270^{\circ}$ azimuth to increase, at least transiently, well beyond the static stall angle of the aerofoil section. This observation is fundamental in explaining the discrepancies between the VTM predictions and the measured forces on the submerged section of the blade that is shown in Figure 8. This is because it suggests that, unlike at the midspan of the blade, those portions of the blade nearest to the tips may indeed be subject to dynamic stall as the blade passes downstream of the axis of rotation of the turbine. Indeed, the discrepancy between the VTM-predicted and measured aerodynamic loading that is shown in Figure 8 is entirely consistent with the presence of dynamic stall, and its characteristic transient overshoot in lift force and reduction in drag on the blades. In contrast, the computational model, with a quasi-static representation of the aerodynamic loads, predicts the reduction in lift and increase in drag that is associated instead with the development of a fully-separated flow on the tip of the blade. This conclusion is underpinned by the data shown in Figure 14, where the integrated load on the lower part of the blade, as predicted by the VTM in conjunction with a dynamic stall model, and the loads on the submerged portion of the blade that were 
measured in the experiment conducted by Strickland et al. [10] are compared. The overall agreement between the experimental measurements and the loads predicted by the VTM in conjunction with a dynamic stall model, particularly in the downstream part of the revolution, is improved considerably compared to the predictions of the same model, but with a quasi-static representation of the blade aerodynamics (see Figure 8).

\section{Wake Structure}

One of the prime features of the Vorticity Transport Model is its ability to portray accurately the evolution of the turbine wake as it develops well downstream of its origin on the blades of the rotor. This is in strong contrast to many other CFD-based techniques where numerical diffusion often results in rapid dissipation of the vorticity in the wake only a short distance downstream of the blades.

Figure 13, introduced earlier, shows a clear contraction of the vertical extent of the wake as the vorticity that is produced by the blades convects downstream of the rotor. On the side of the rotor where the ground plane is modelled, the angle between the vortex trajectory and the ground plane is smaller compared to the corresponding angle of the trajectory of the vortices trailed by the upper blade tip. Furthermore, it is apparent that self-induction causes the vortices on the side of the rotor where the ground plane is present to travel faster downstream than the vortices that are trailed from the upper blade tip. This results in a skewing of the wake such that the equivalent tip vortices interact earlier with the lower tip of the blade at $270^{\circ}$ azimuth than the upper, as is shown clearly in Figure 13. This observation may have consequences for the unsteady torsional and bending loads in the support structure of vertical-axis wind turbines that are operated close to the ground.

Figure 15 shows a rendition of the overall, three-dimensional structure of the wake, visualised as a surface within the flow on which the vorticity in the wake has constant magnitude. The vorticity from each blade is rendered in a separate shade. Figure 15(a) demonstrates the complexity of the evolution of the wake downstream of the turbine - the essentially cycloidal structure of the initial sections of the wake is seen to break down relatively rapidly as the self-induced velocity of both the shed and trailed vorticity produced by the blades acts to distort and disorder the wake into a complicated tangle of individual vortical filaments. The interaction between the lower tip of the reference blade ('blade 1') and the vorticity in the wake is shown in detail in the inset to Figure 15(b), and reveals that two different types of blade-vortex interactions are present within the system. Indeed, a complex, intermeshed system of vorticity is revealed in which interactions between the reference blade and its own tip vortex trailed in a previous revolution of the turbine as well as an interaction between the reference blade and the tip vortex trailed by the other blade of the turbine are both present. Figure 15 certainly supports the conclusion reached by previous researchers in the field, such as Klimas [2] amongst others, that the accurate modelling of the wake of such systems poses one of the greatest challenges that must be overcome before the correct portrayal of their aerodynamic performance will become a possibility. 


\section{Conclusion}

The flow field around a Darrieus-type vertical-axis wind turbine has been predicted numerically using Brown's Vorticity Transport Model (VTM). The VTM-predicted forces for an aerofoil section at the mid-span of the blades of the turbine compare very well with previous CFD simulations of the two-dimensional aerodynamics of the same aerofoil in an equivalent planar, cyclic motion. Since the presence of dynamic stall for the simulated tip speed ratio was discounted in previous studies, the effect of dynamic stall was not explicitly modelled in initial VTM simulations. The loads that were predicted by these initial VTM simulations compare very favourably with experimental measurements of the loading on the blades of the turbine, except when the blade is immediately downstream of the axis of rotation of the rotor. In the downstream part of the revolution of the turbine, aerodynamic interactions occur between the blades of the rotor and vortices in the wake. These blade-wake interactions cause transient excursions in the angle of attack of the blade beyond the static stall angle, leading to localised dynamic stall near the tips of the blades. Indeed, the conclusion that local dynamic stall is induced by blade-wake interactions is supported by the improved correlation between experimental measurements and VTM predictions that result when a dynamic stall model is incorporated into the VTM to represent the blade aerodynamics compared to when only a quasi-static model was used. It is suggested, thus, that the disagreement between experimental and numerical data that has been presented in previous studies arises because three-dimensional effects, such as interactions between the blades of the turbine and the vortices in the wake, were not modelled with sufficient fidelity.

The VTM provides detailed insight into the structure of the vortical wake developed by the turbine, and shows clearly the emergence of strong three-dimensional effects in the flow near the rotor that are mediated by the dynamics of the vorticity that is shed and trailed into the wake behind the turbine. This paper contributes to an understanding of the flow field surrounding vertical-axis wind turbines and suggests strongly that accurate modelling of the structure of the turbine wake and the associated three-dimensional effects on the blade loading is an essential precursor to high fidelity prediction of the aerodynamic performance of such devices. 


\section{References}

1. Darrieus GJM. Turbine having its rotating shaft transverse to the flow of the current. US Patent 1,835,018. December 1931.

2. Klimas, PC. Darrieus rotor aerodynamics. Transactions of the ASME. Journal of Solar Energy Engineering 1982; 104: 102-105.

3. Strickland JH. The Darrieus turbine: a performance prediction model using multiple streamtubes. Sandia Technical Report SAND75-0431, 1975.

4. Paraschivoiu I. Double-multiple streamtube model for studying vertical-axis wind turbines. Journal of Propulsion and Power 1988; 4: 370-377.

5. Strickland JH, Webster BT, Nguyen, T. A vortex model of the Darrieus turbine: an analytical and experimental study. Transactions of the ASME. Journal of Fluids Engineering 1979; 101: 500-505.

6. Hirsch H, Mandal AC. A cascade theory for the aerodynamic performance of Darrieus wind turbines. Wind Engineering 1987; 11: 164-175.

7. Hansen MOL, Sørensen DN. CFD model for vertical axis wind turbine. Wind Energy for the New Millennium - Proceedings of the European Wind Energy Conference, Copenhagen, $2001 ; 485-488$.

8. Simão Ferreira CJ, van Bussel G, van Kuik G. 2D CFD simulation of dynamic stall on a vertical axis wind turbine: verification and validation with PIV measurements. 45th AIAA Aerospace Sciences Meeting and Exhibit/ASME Wind Energy Symposium, Reno 2007; 1619116201.

9. Horiuchi K, Ushiyama I, Seki K. Straight wing vertical axis wind turbines: a flow analysis. Wind Engineering 2005; 29: 243-252.

10. Strickland JH, Smith T, Sun K. A vortex model of the Darrieus turbine: an analytical and experimental study. Sandia Technical Report SAND81-7017, 1981.

11. Ponta FL, Jacovkis PM. A vortex model for Darrieus turbine using finite element techniques. Renewable Energy 2001; 24: 1-18.

12. Brown RE. Rotor wake modelling for flight dynamic simulation of helicopters. AIAA Journal 2000; 38: 57-63.

13. Brown RE, Line AJ. Efficient high-resolution wake modelling using the vortex transport equation. AIAA Journal 2005; 43: 1434-1443.

14. Laneville A, Vittecoq P. Dynamic stall: the case of the vertical axis wind turbine. Transactions of the ASME. Journal of Solar Energy Engineering 1986; 108: 140-145.

15. Leishman JG, Beddoes TS. A semi-empirical model for dynamic stall. Journal of the American Helicopter Society 1989; 34: 3-17.

16. Gupta S, Leishman JG. Dynamic stall modelling of the S809 aerofoil and comparison with experiments. Wind Energy 2006; 9: 521-547. 
17. Beddoes TS. A third generation model for unsteady aerodynamics and dynamic stall. Westland Helicopters Ltd., RP-908, 1993.

18. Sheng, W., Galbraith, R.A.McD and Coton, F.N. A modified dynamic stall model for low Mach numbers. In Proceedings of the 45th AIAA Aerospace Sciences Meeting and Exhibit, Reno, Nevada, USA, 2007.

19. Niven AJ, Galbraith RAMcD. Modelling dynamic stall vortex inception at low Mach numbers. Aeronautical Journal 1997; 101: 67-76.

20. Toro E. A weighted average flux method for hyperbolic conservation laws. Proceedings of the Royal Society of London, Series A: Mathematical and Physical Sciences 1981; 423: 401418.

21. Fletcher TM, Brown RE, Kim DH, Kwon OJ. Predicting wind turbine blade loads using vorticity transport and RANS methodologies. European Wind Energy Conference, Marseille 2009.

22. Hand MM, Simms DA, Fingersh LJ, Jager DW, Cotrell JR, Schreck S, Larwood SM. Unsteady aerodynamics experiment phase VI: wind tunnel test configurations and available data campaigns. NREL Technical Report NREL/TP-500-29955, 2001.

23. Fletcher TM, Brown RE. Simulating wind turbine interactions using the vorticity transport model. 47th AIAA Aerospace Sciences Meeting and Exhibit/ASME Wind Energy Symposium, Orlando 2009.

24. Tangler JL. The nebulous art of using wind tunnel aerofoil data for predicting rotor performance. Wind Energy 2002; 5: 245-257.

25. Althaus D. Profilpolaren für den Modellflug. Neckar-Verlag: Villingen-Schwenningen, 1980.

26. Drela M. XFoil: an analysis and design system for low Reynolds number airfoils (XFOIL 6.94, 18 December 2001). in Mueller TJ (ed.). Low Reynolds number aerodynamics. Lecture Notes in Engineering; 54:1-12, Springer-Verlag: Berlin, 1989.

27. Abbott IH, von Doenhoff, AE. Theory of wing sections: including a summary of airfoil data. Dover Publications, New York, 1959. 
Table 1: Rotor parameters

\begin{tabular}{lr}
\hline \hline & \\
Number of blades & 2 \\
Aerofoil section & NACA 0012 \\
Average blade Reynolds number & 40,000 \\
Aerofoil chord length & $9.14 \mathrm{~cm}$ \\
Rotor tip speed & $45.7 \mathrm{~cm} / \mathrm{s}$ \\
Chord-to-radius ratio & 0.15 \\
Tip speed ratio & 5 \\
\end{tabular}




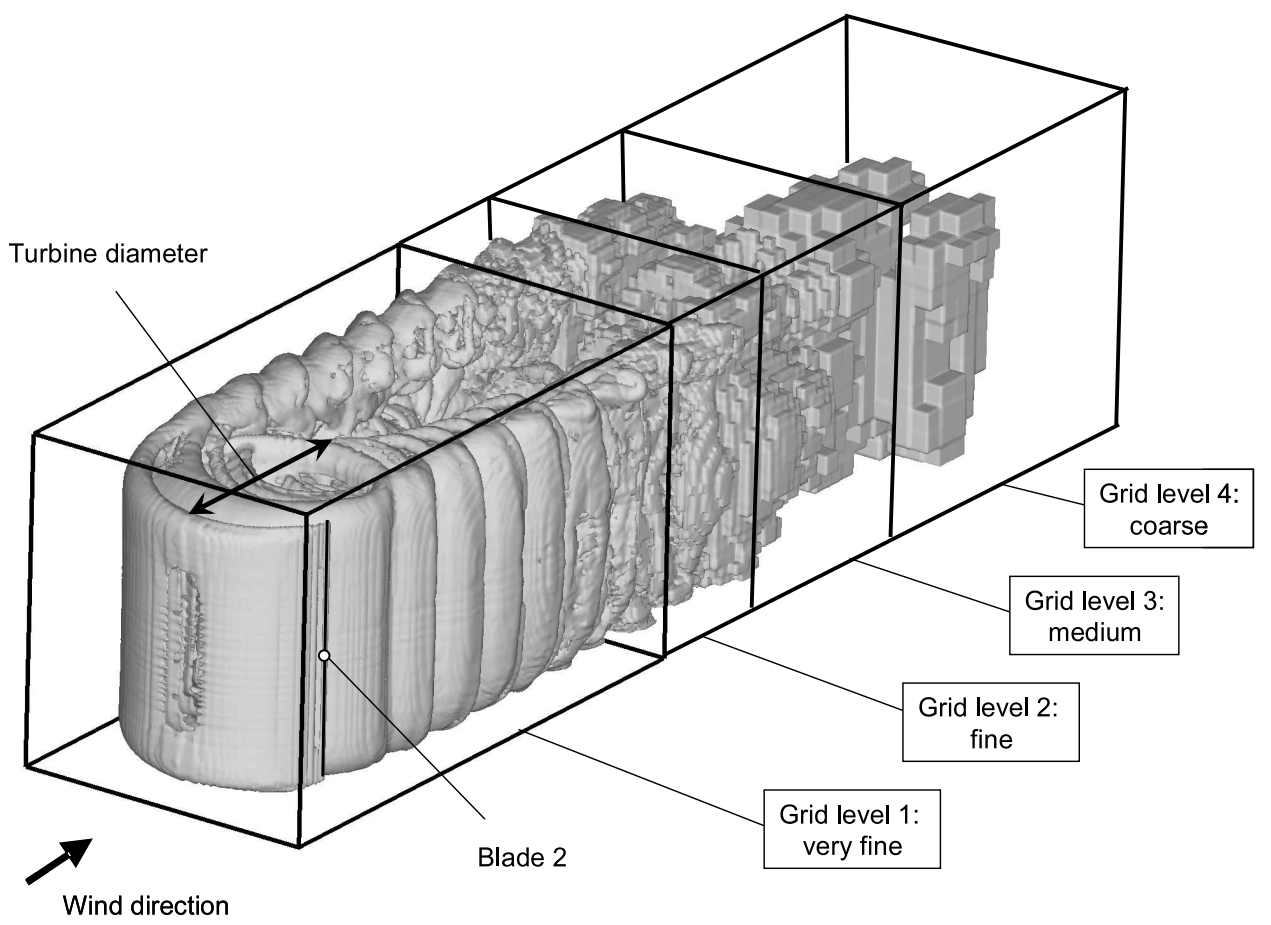

Figure 1: Computed vorticity in nested grid boxes that contain cells of different size; snapshot after 7 turbine revolutions. 


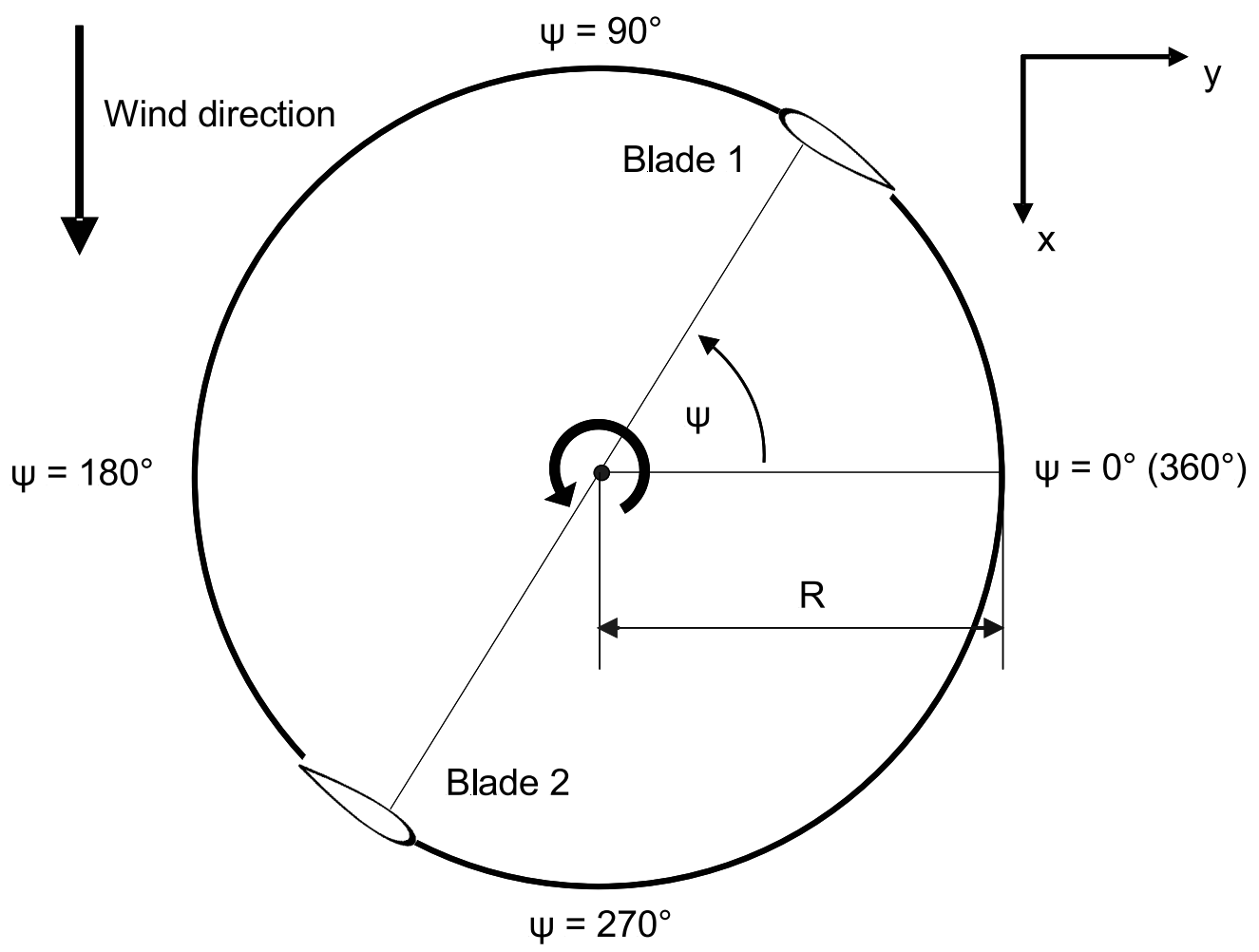

Figure 2: Orientation of the blades and specification of the azimuth angle. 


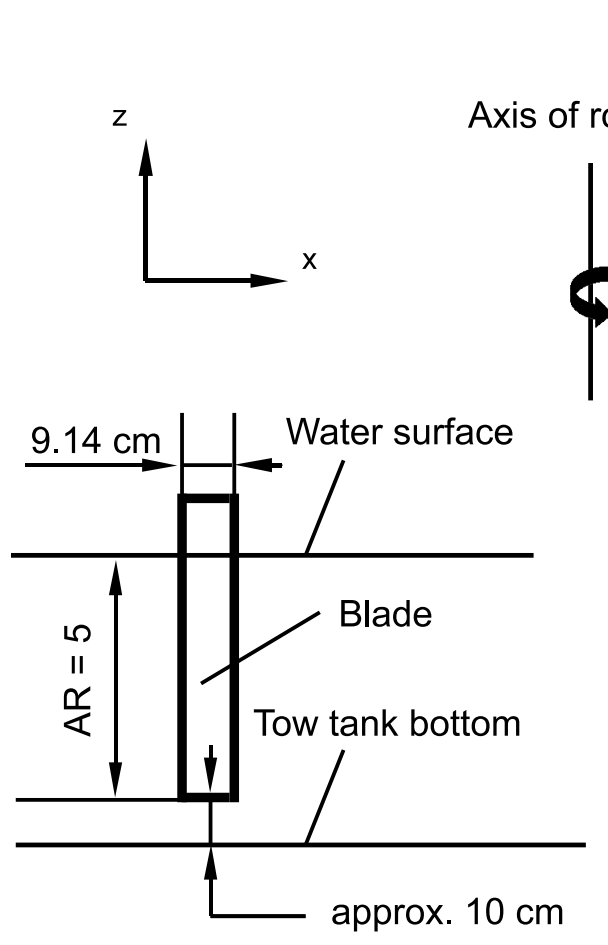

(a)

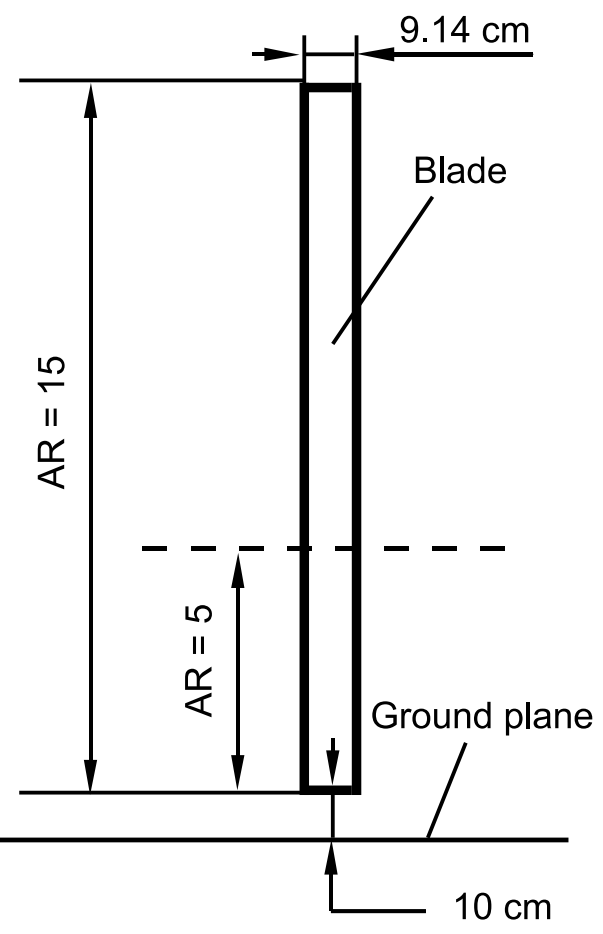

(b)

Figure 3: Experimental arrangement (a), and its computational representation (b). 


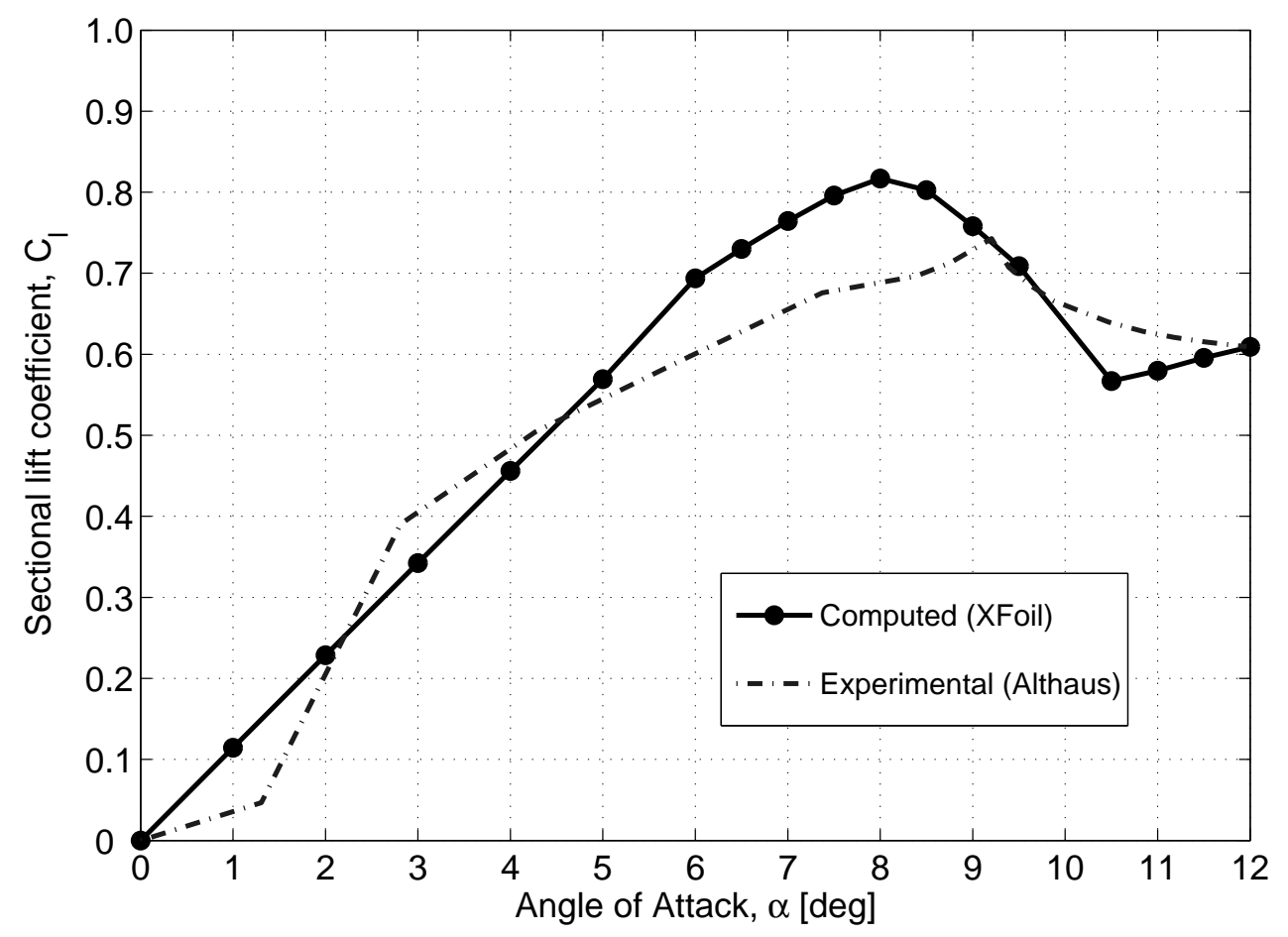

Figure 4: Variation of sectional lift coefficient with angle of attack on the NACA 0012 aerofoil $(R e=40,000)$. 


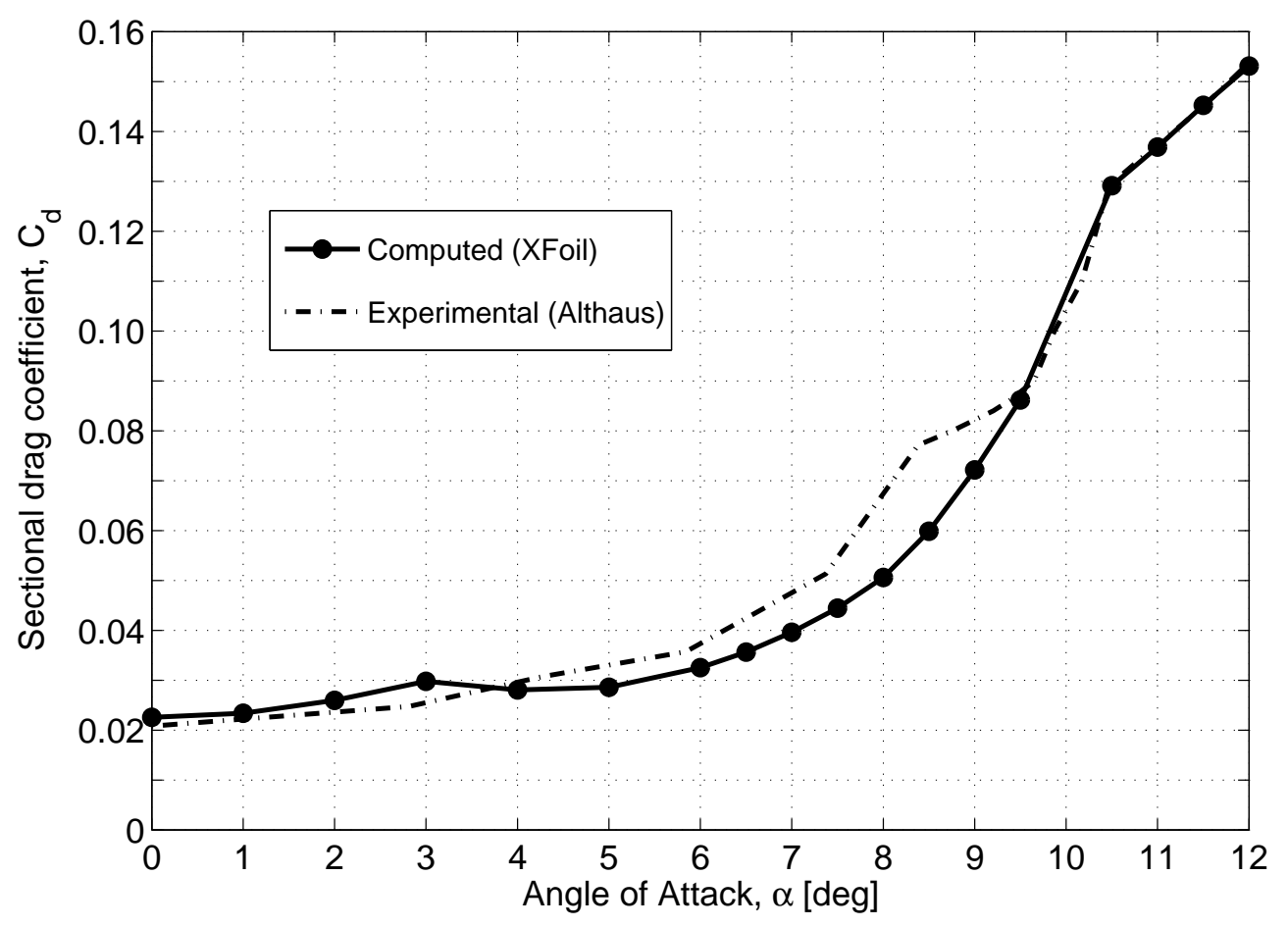

Figure 5: Variation of sectional drag coefficient with angle of attack on the NACA 0012 aerofoil $(R e=40,000)$. 


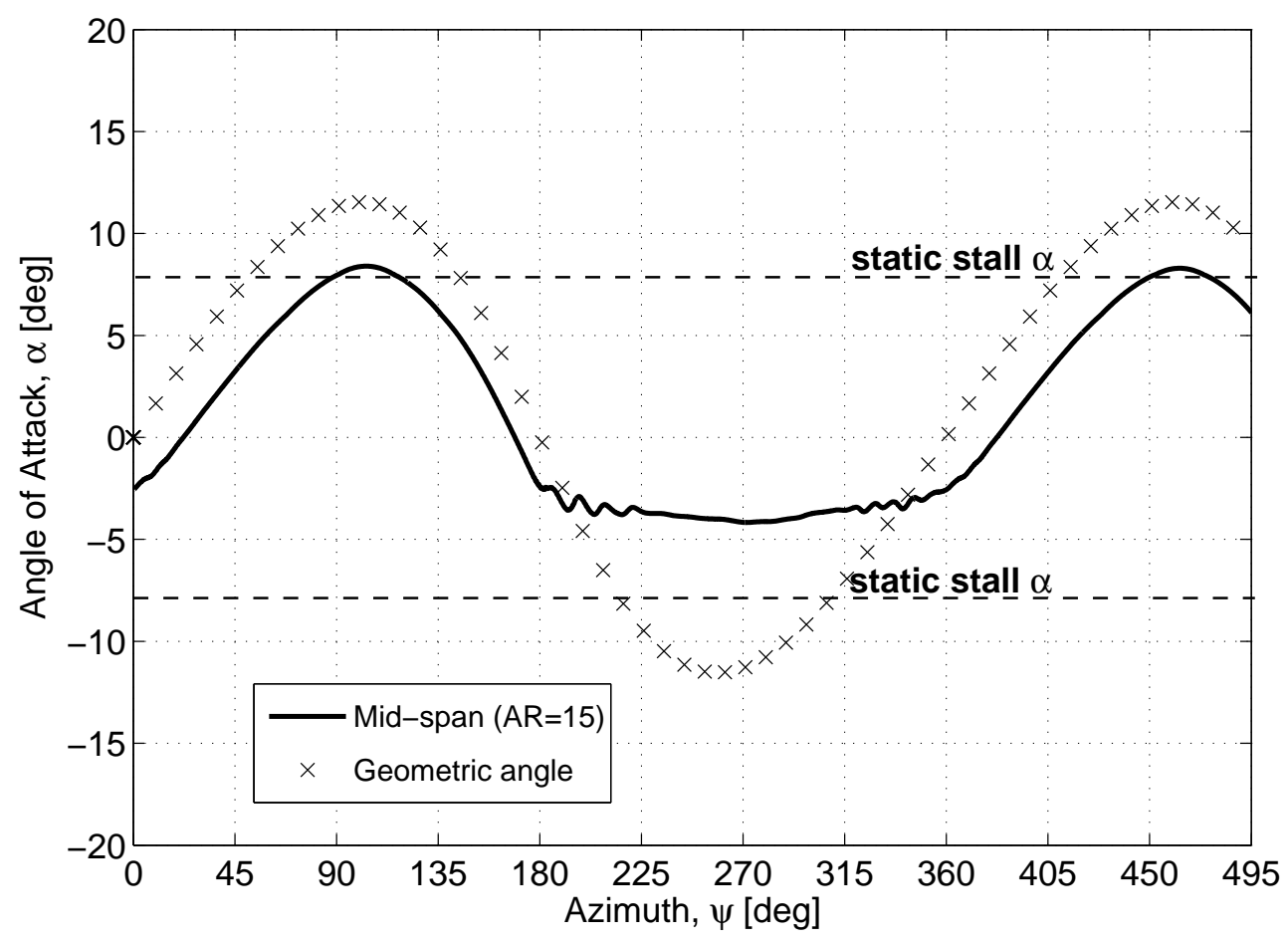

Figure 6: Comparison between the VTM-simulated variation of aerodynamic angle of attack with blade azimuth (XFoil data) for the section at mid-span of the blade and the geometric angle of attack given by Equation 8 (i.e in the absence of induced effects). 


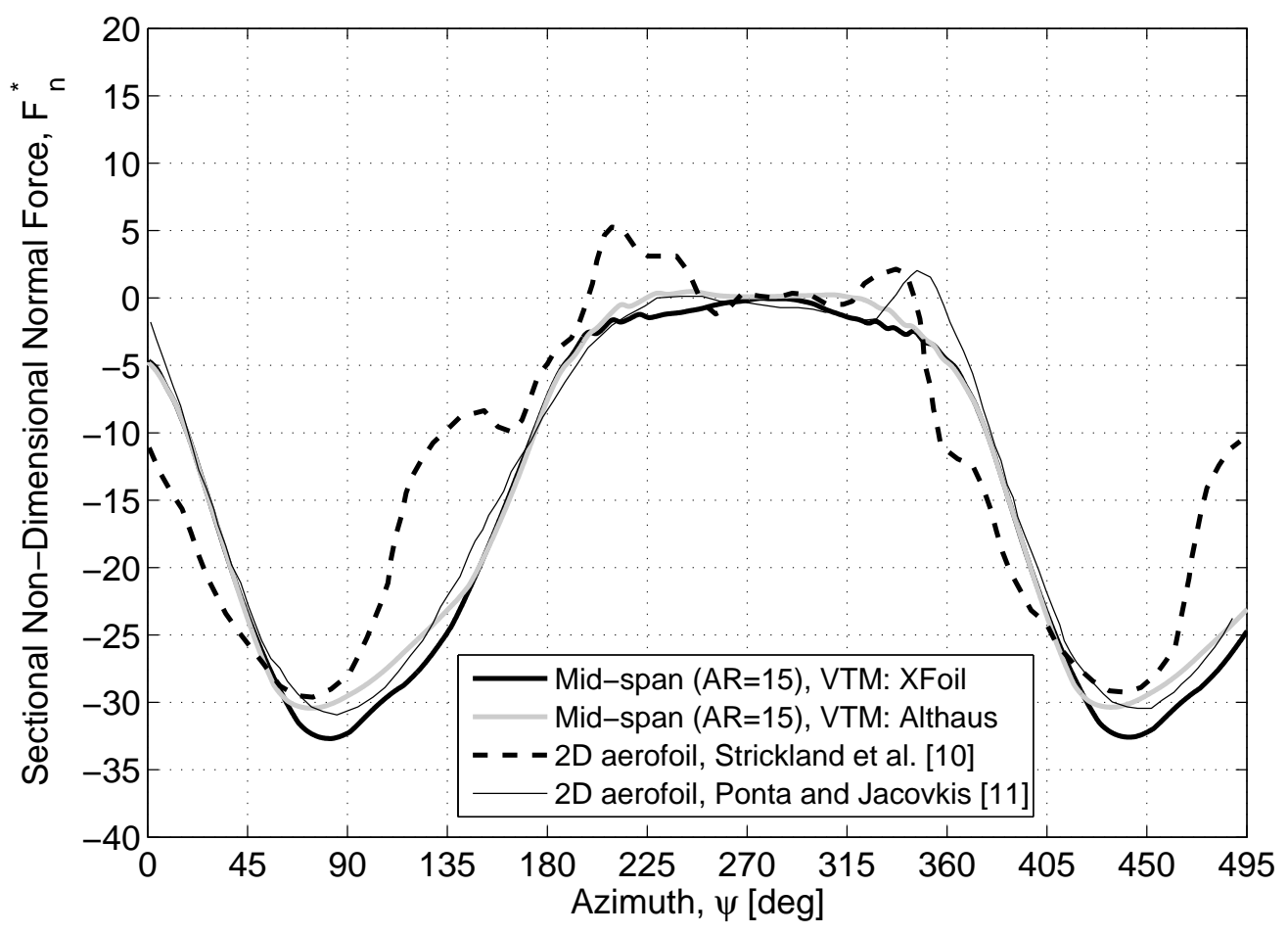

(a) Normal force

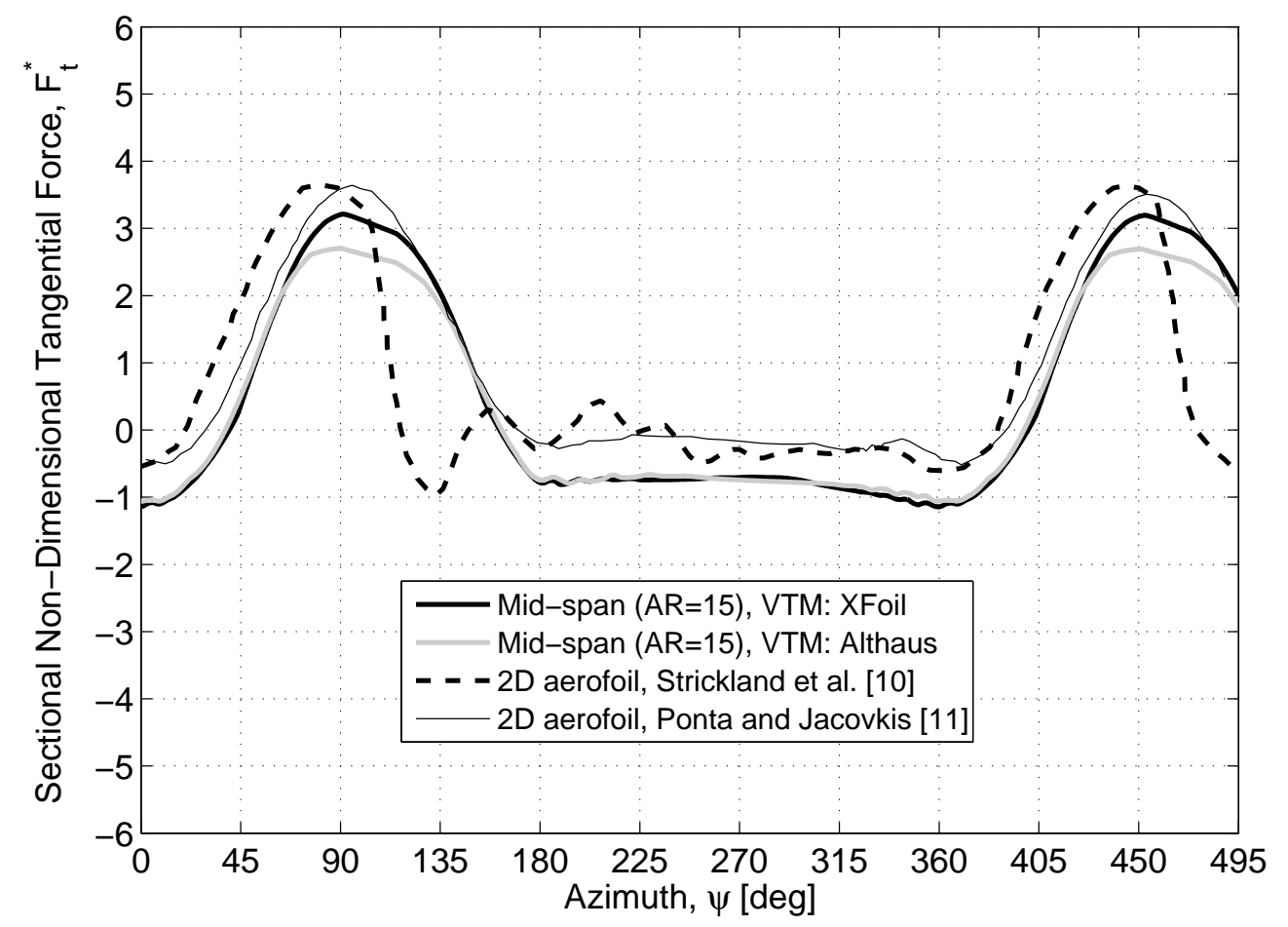

(b) Tangential force

Figure 7: Variation in VTM-predicted non-dimensional normal force (a), and non-dimensional tangential force (b) with blade azimuth for the section at mid-span of the blade compared to CFD simulations of the $2 D$ aerodynamics of the same aerofoil in a planar cyclic motion. 


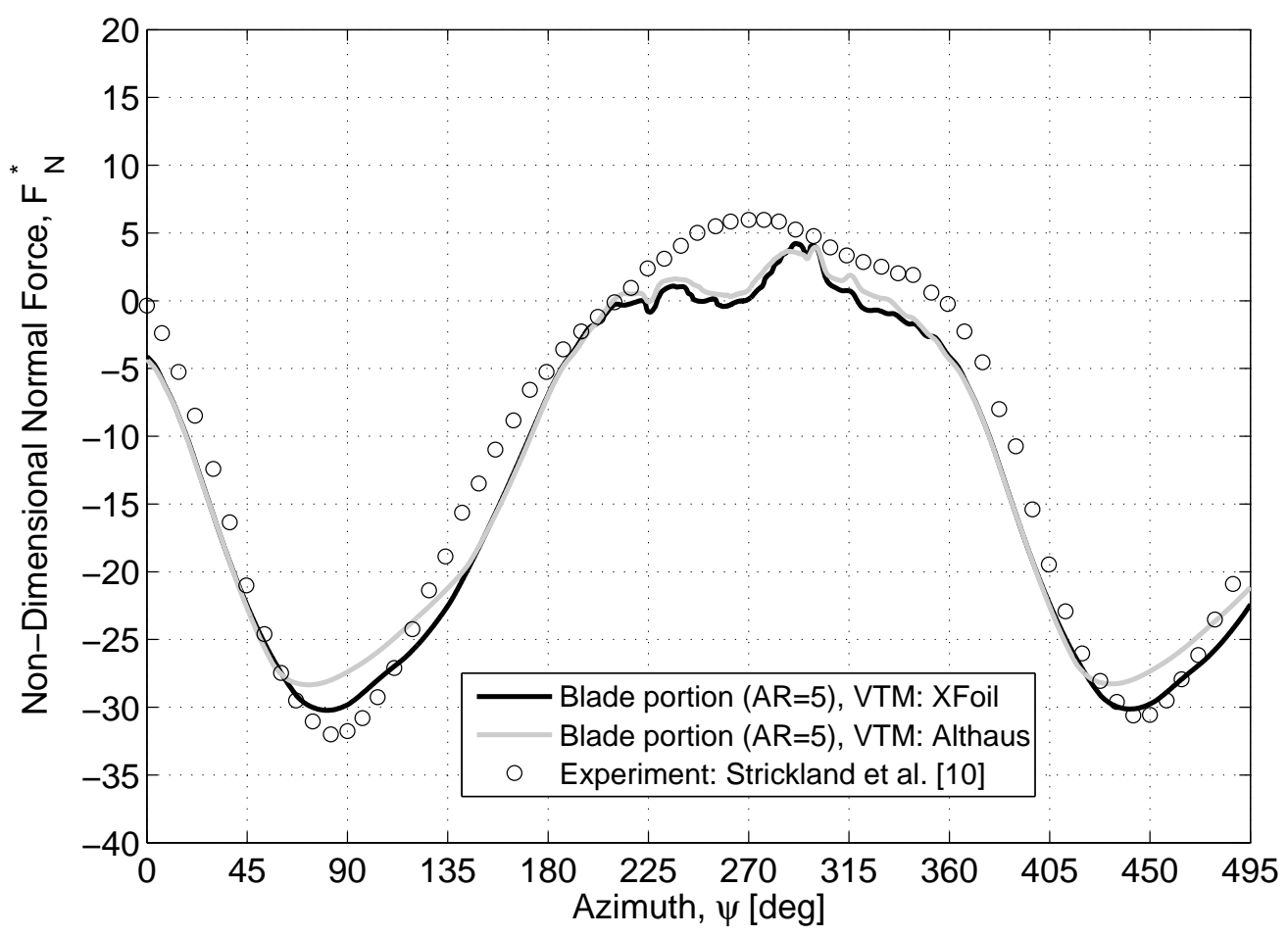

(a) Normal force

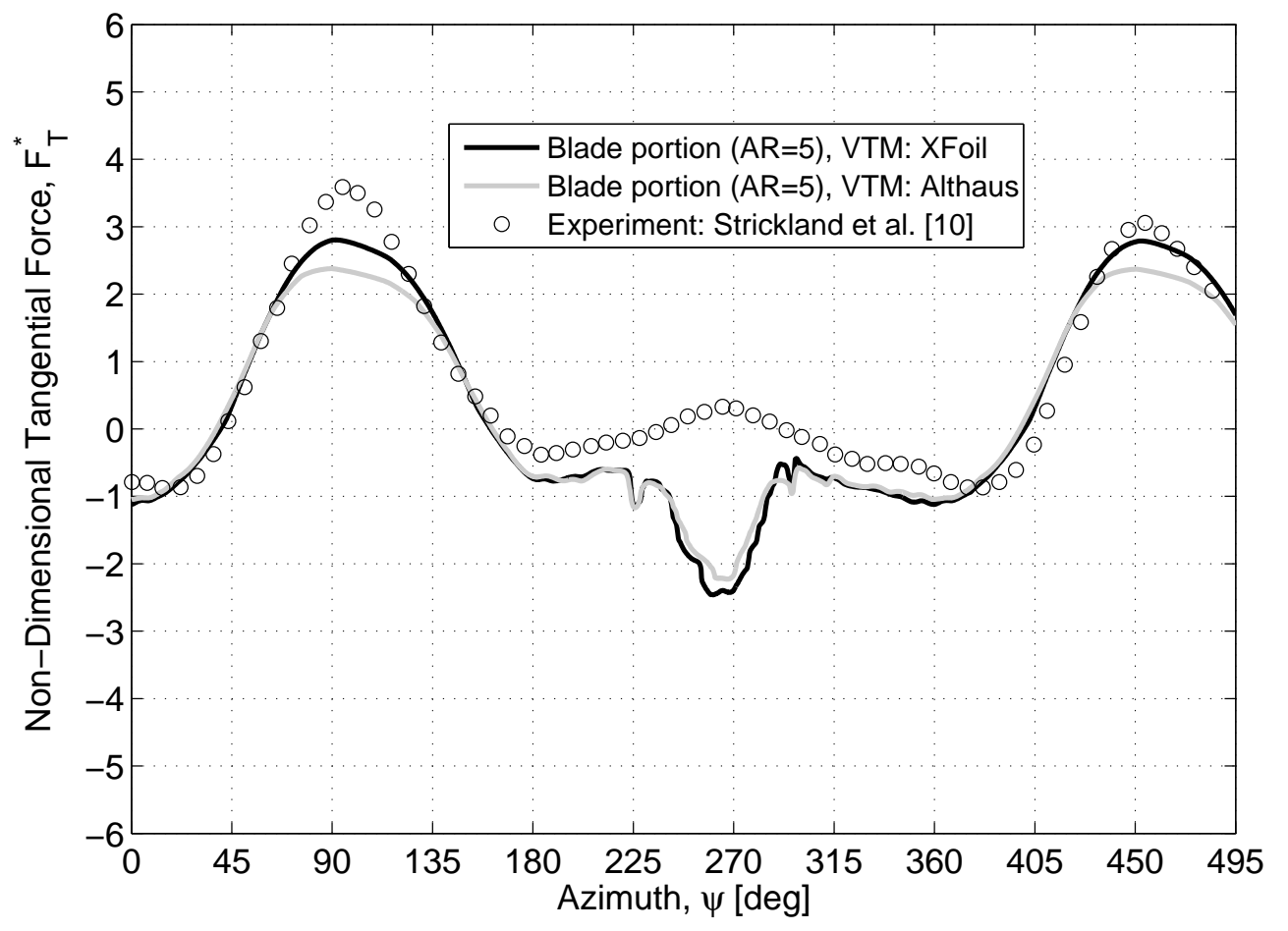

(b) Tangential force

Figure 8: Comparison between the variation in VTM-predicted and measured non-dimensional normal force (a), and non-dimensional tangential force (b). No dynamic stall model used in the analysis. 


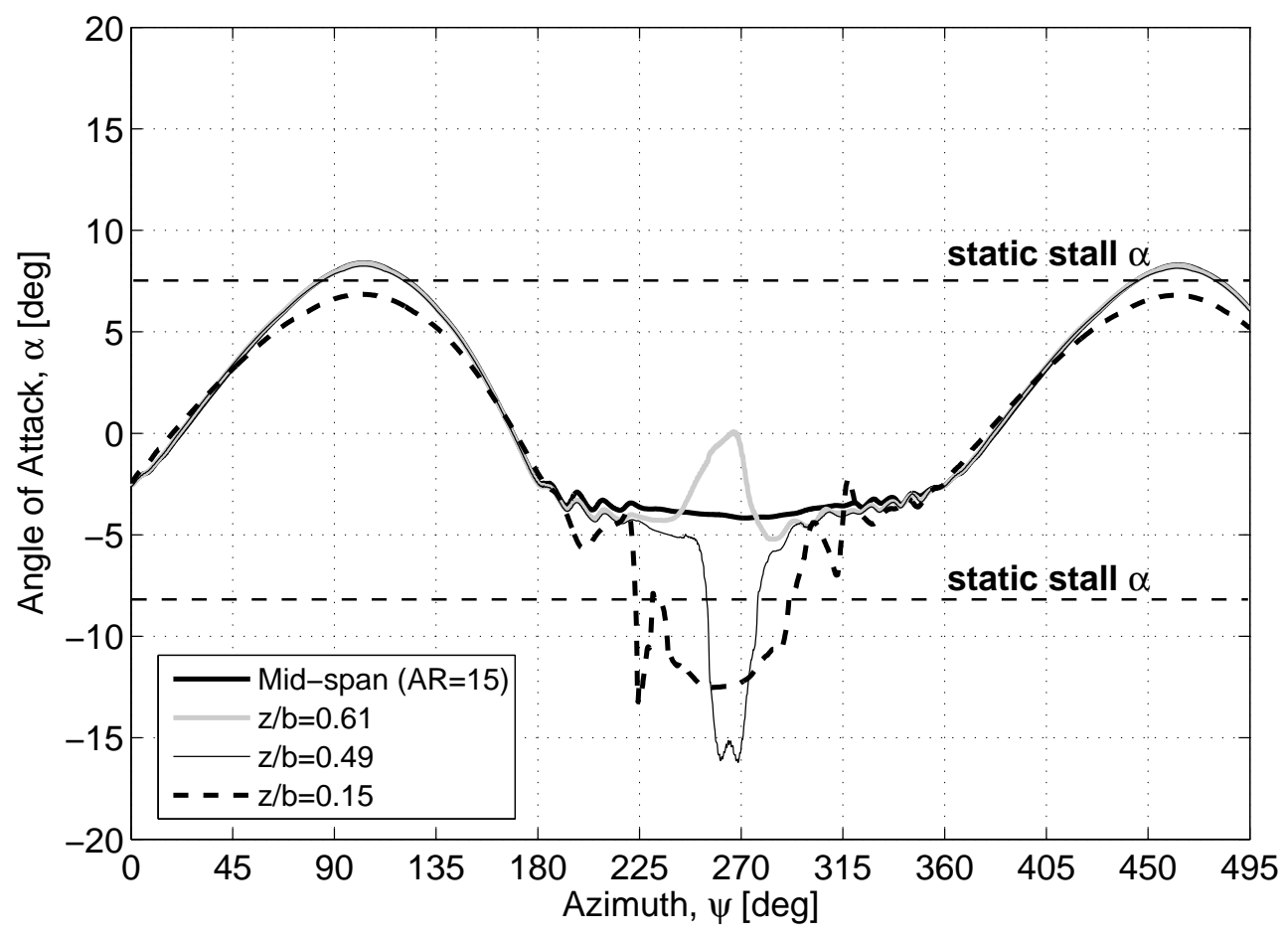

Figure 9: VTM-predicted variation in aerodynamic angle of attack with blade azimuth at the section at mid-span of the blade with aspect ratio 15, and at three blade sections located along the lower part of the blade (XFoil data). No dynamic stall model used in the analysis. 


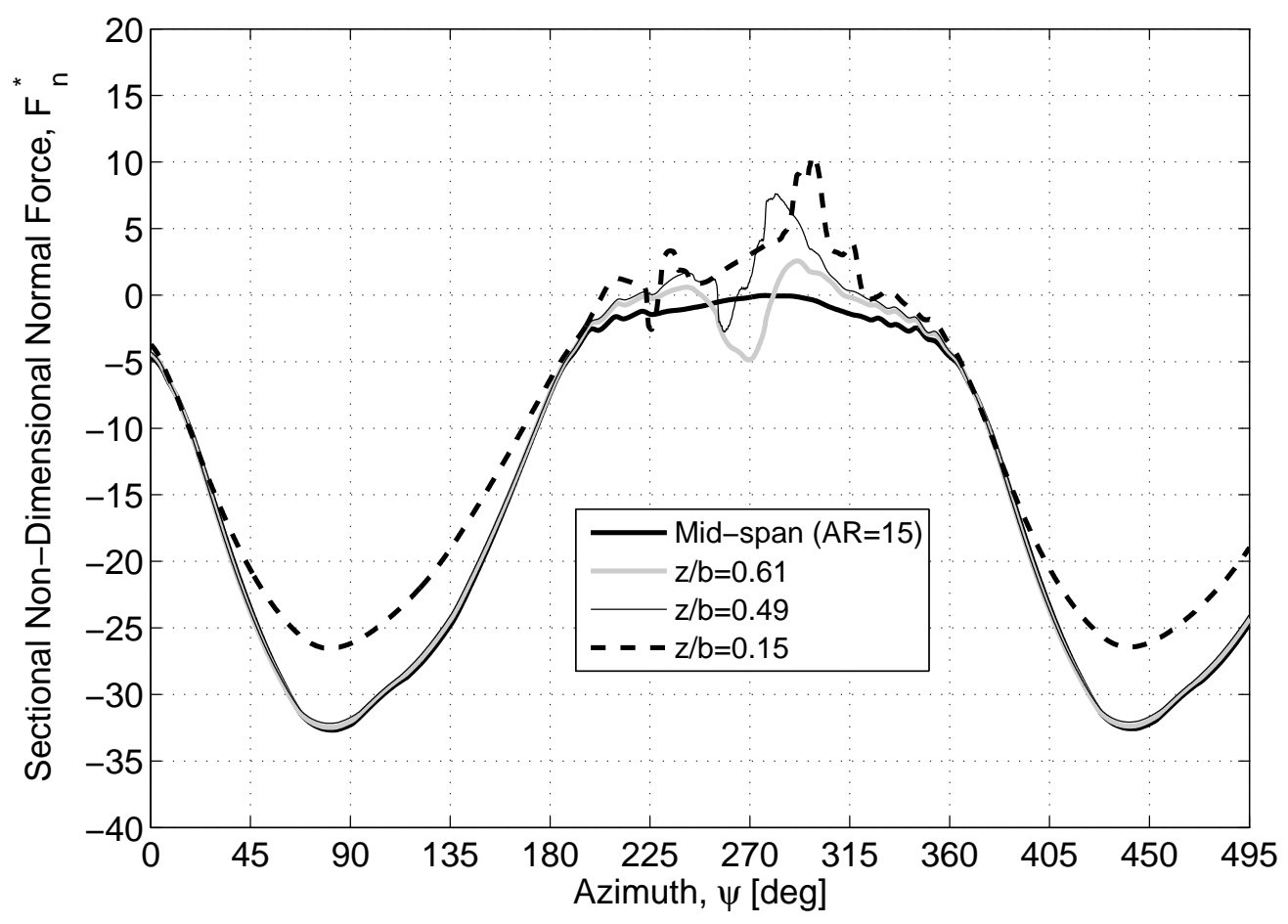

(a) Normal force

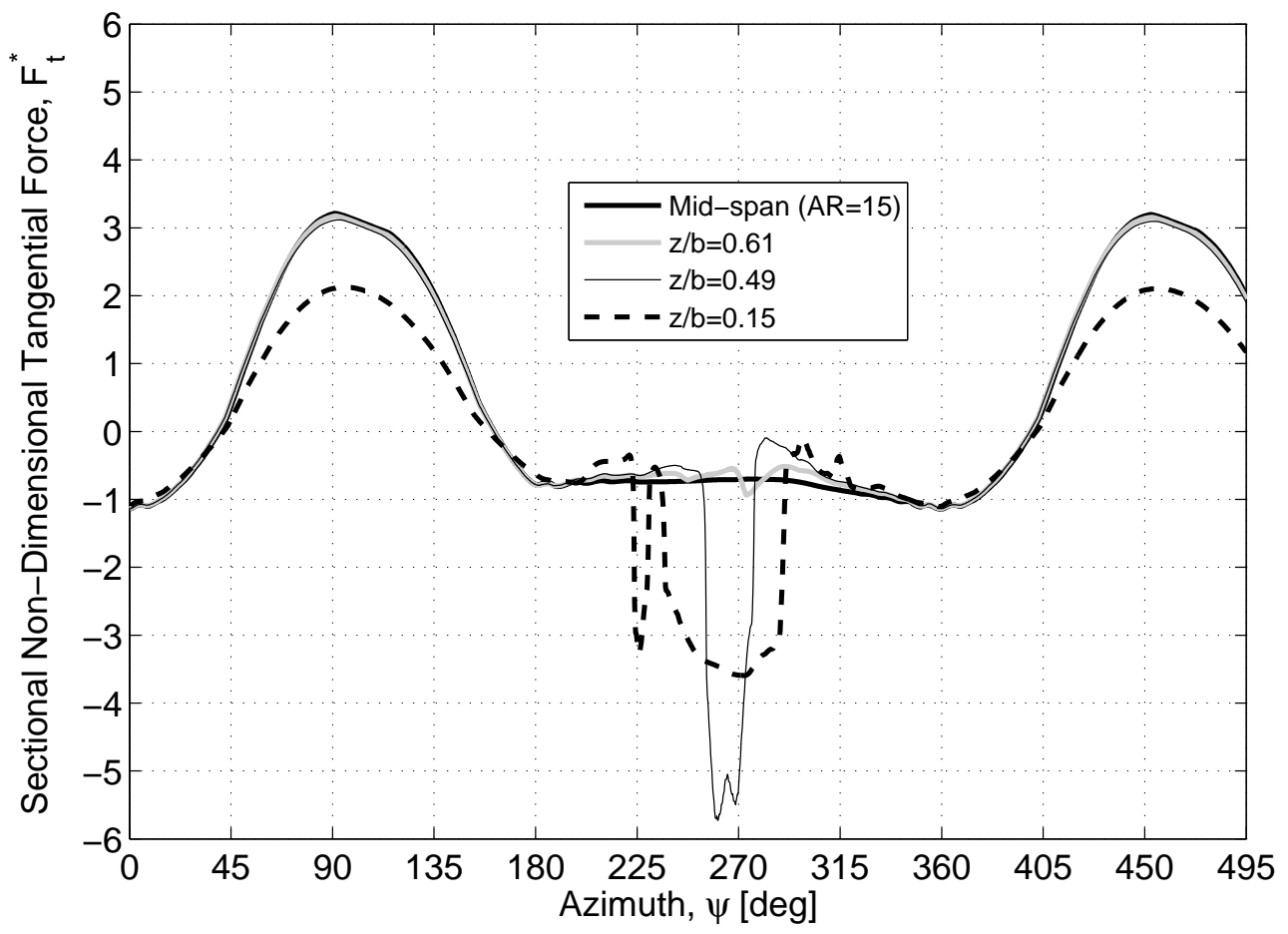

(b) Tangential force

Figure 10: VTM-predicted variation in sectional non-dimensional normal force (a), and sectional non-dimensional tangential force (b) with blade azimuth at the section at mid-span of the blade with aspect ratio 15, and at three blade sections located along the lower part of the blade (XFoil data). No dynamic stall model used in the analysis. 


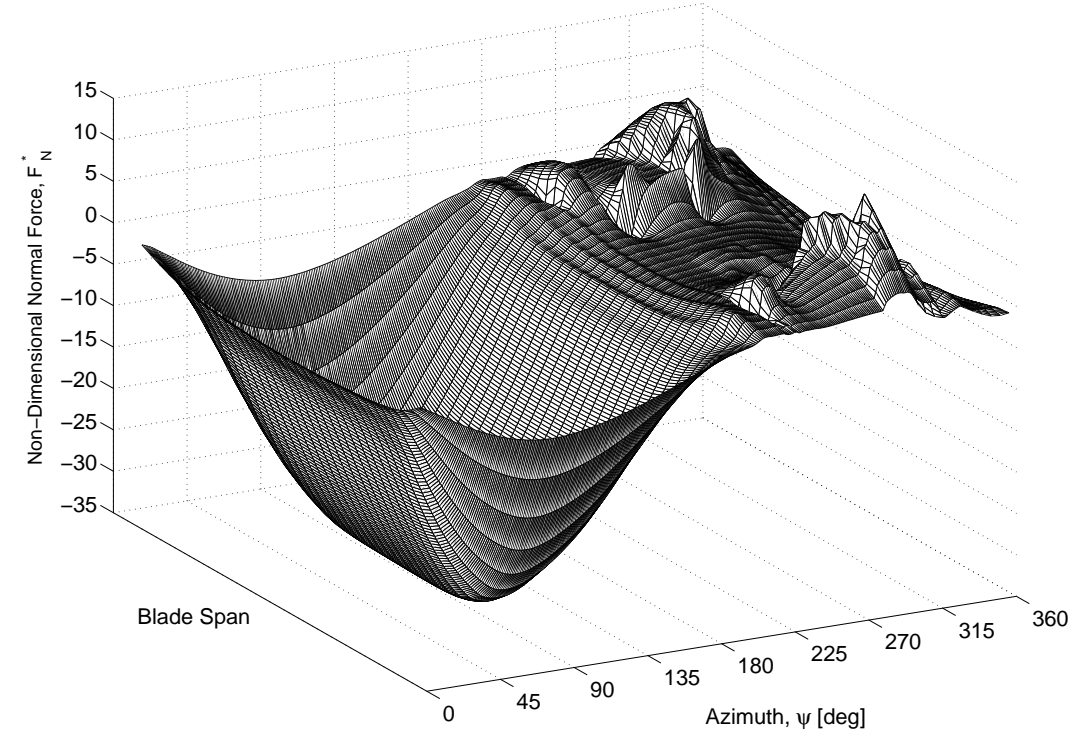

(a) Visulisation of unsteady normal force distribution

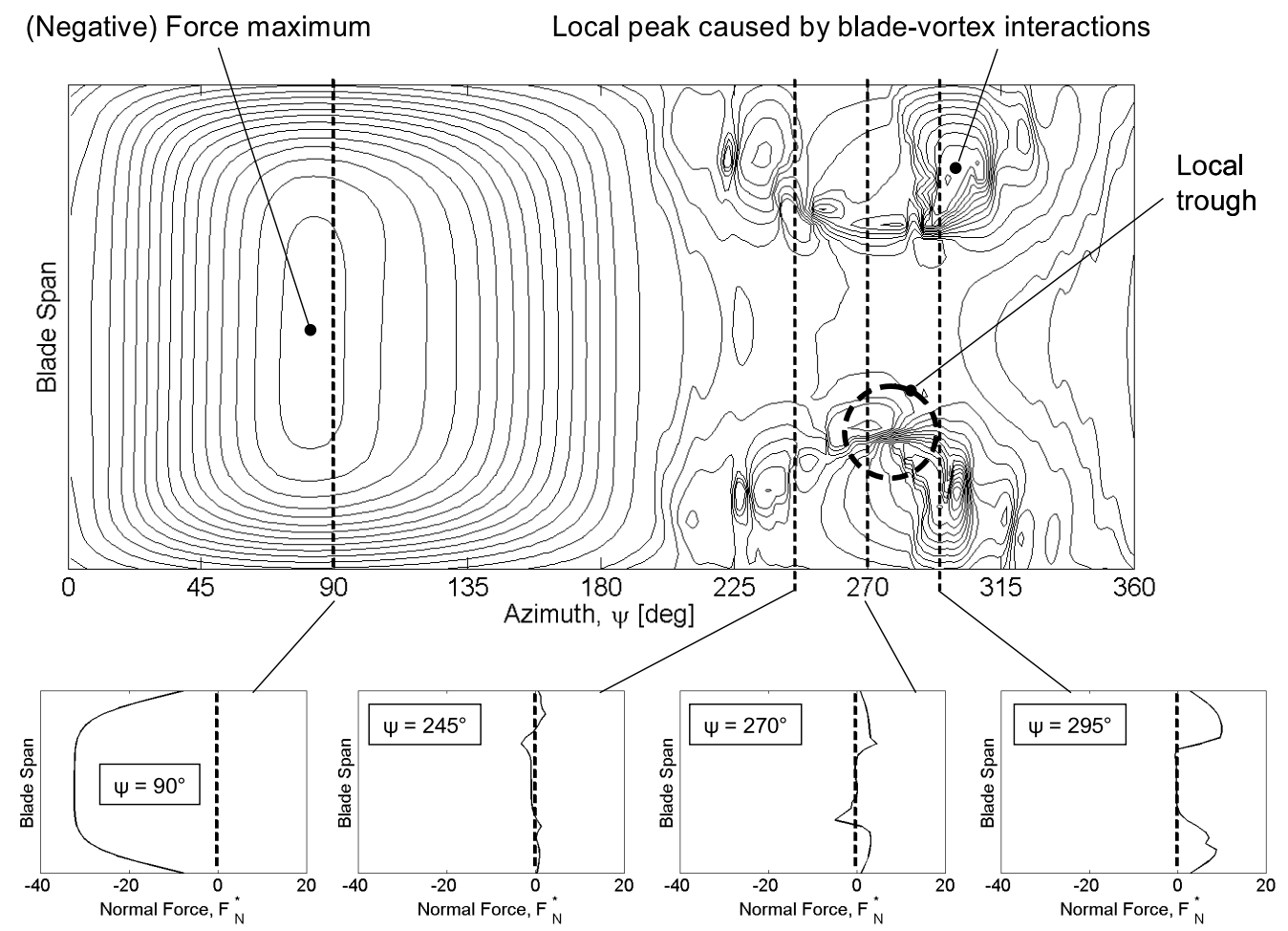

(b) Contours and line plot of unsteady normal force

Figure 11: VTM-predicted variation with azimuth of the normal force along the blade span. 


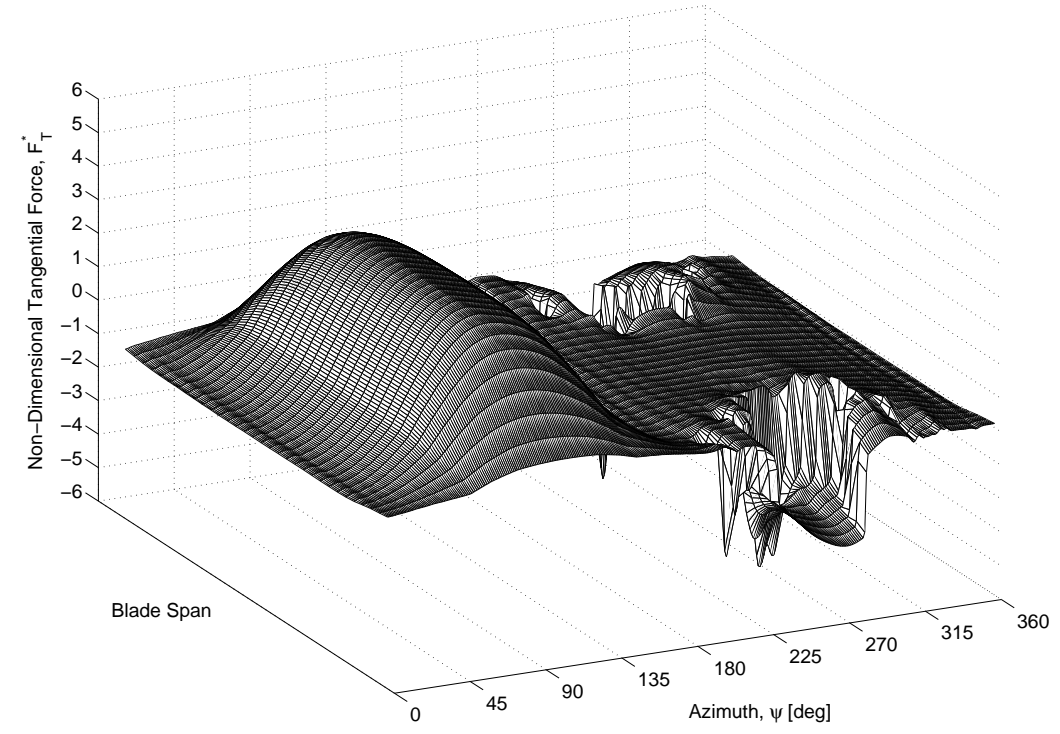

(a) Visulisation of unsteady tangential force distribution

Note uniform distribution between $0^{\circ}-180^{\circ}$ and impulsive perturbations between $180^{\circ}-360^{\circ}$ azimuth

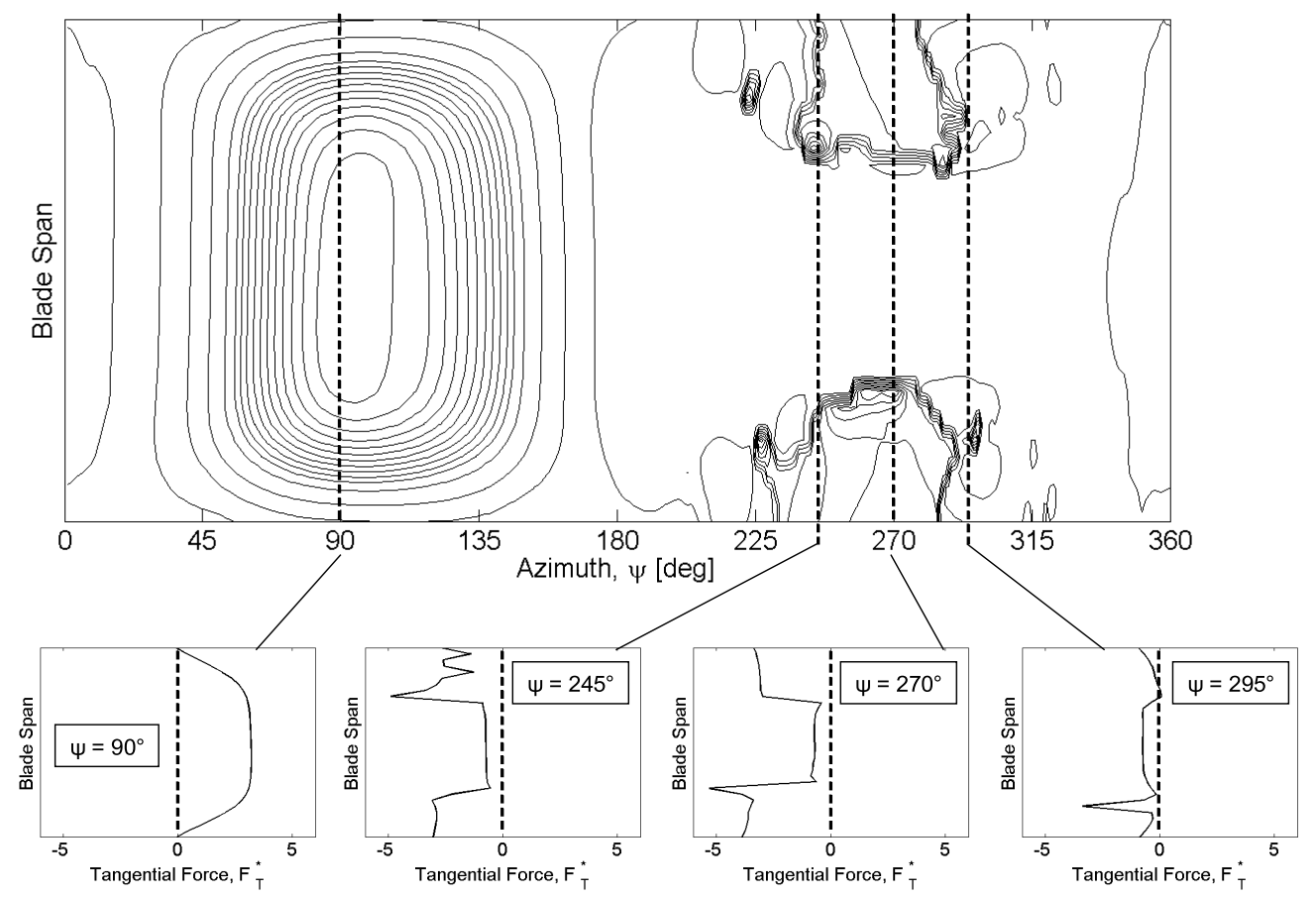

(b) Contours and line plot of unsteady tangetial force

Figure 12: VTM-predicted variation with azimuth of the tangential force along the blade span. 


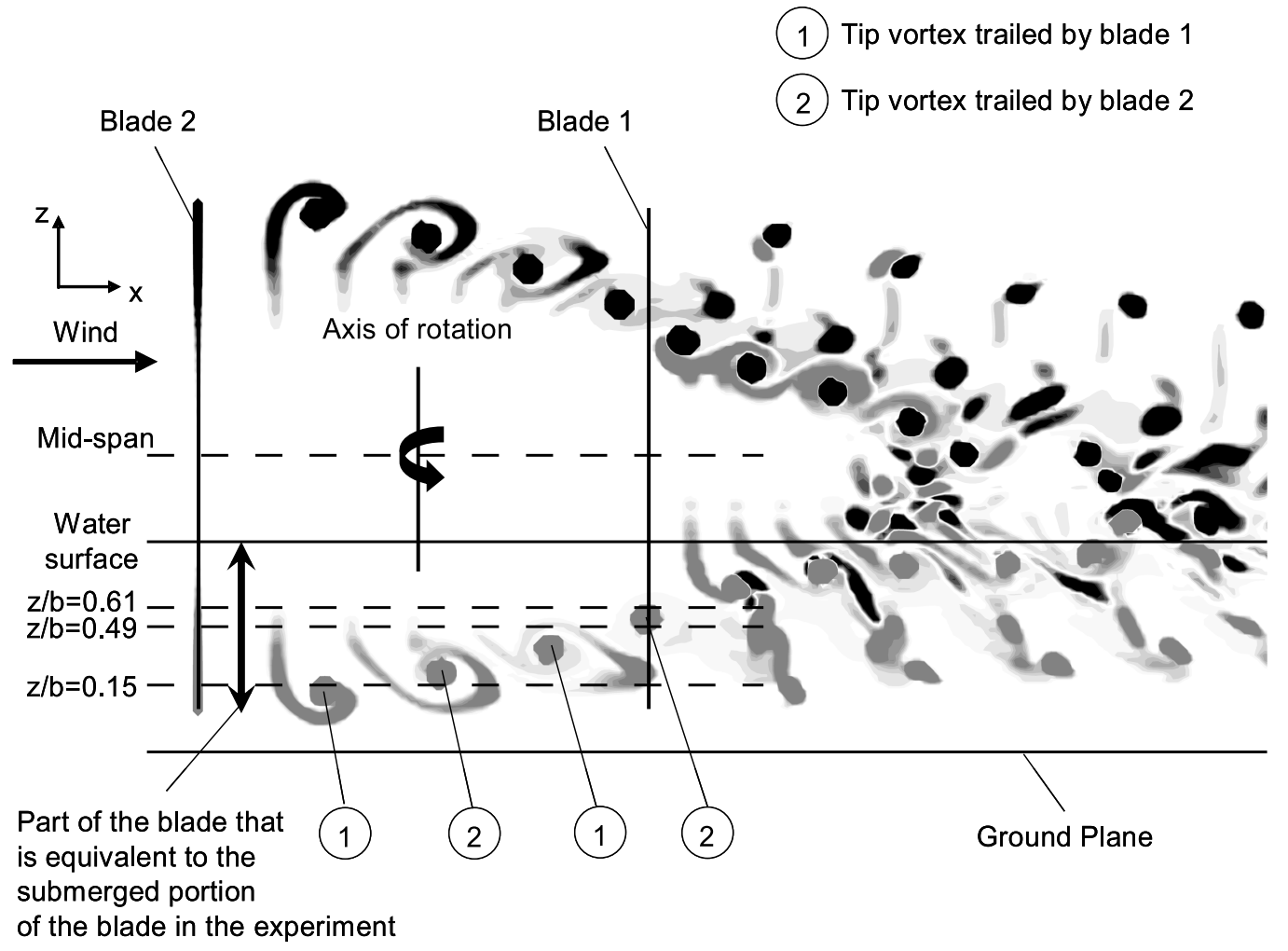

Figure 13: Computed vorticity field surrounding the turbine, represented using contours of vorticity on a vertical plane containing the axis of rotation of the turbine. Blade 1 is located at azimuth $\psi=270^{\circ}$. 


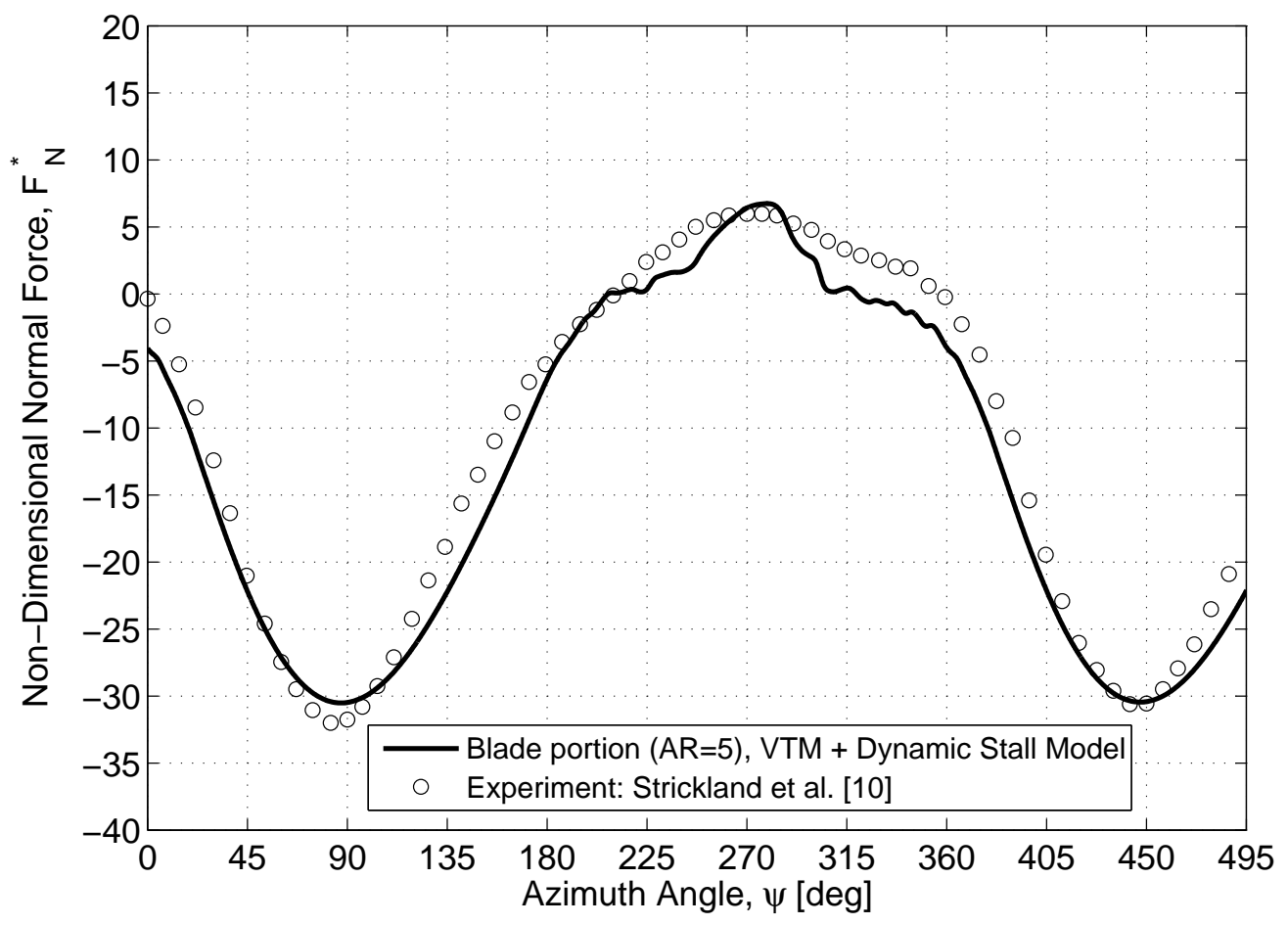

(a) Normal force

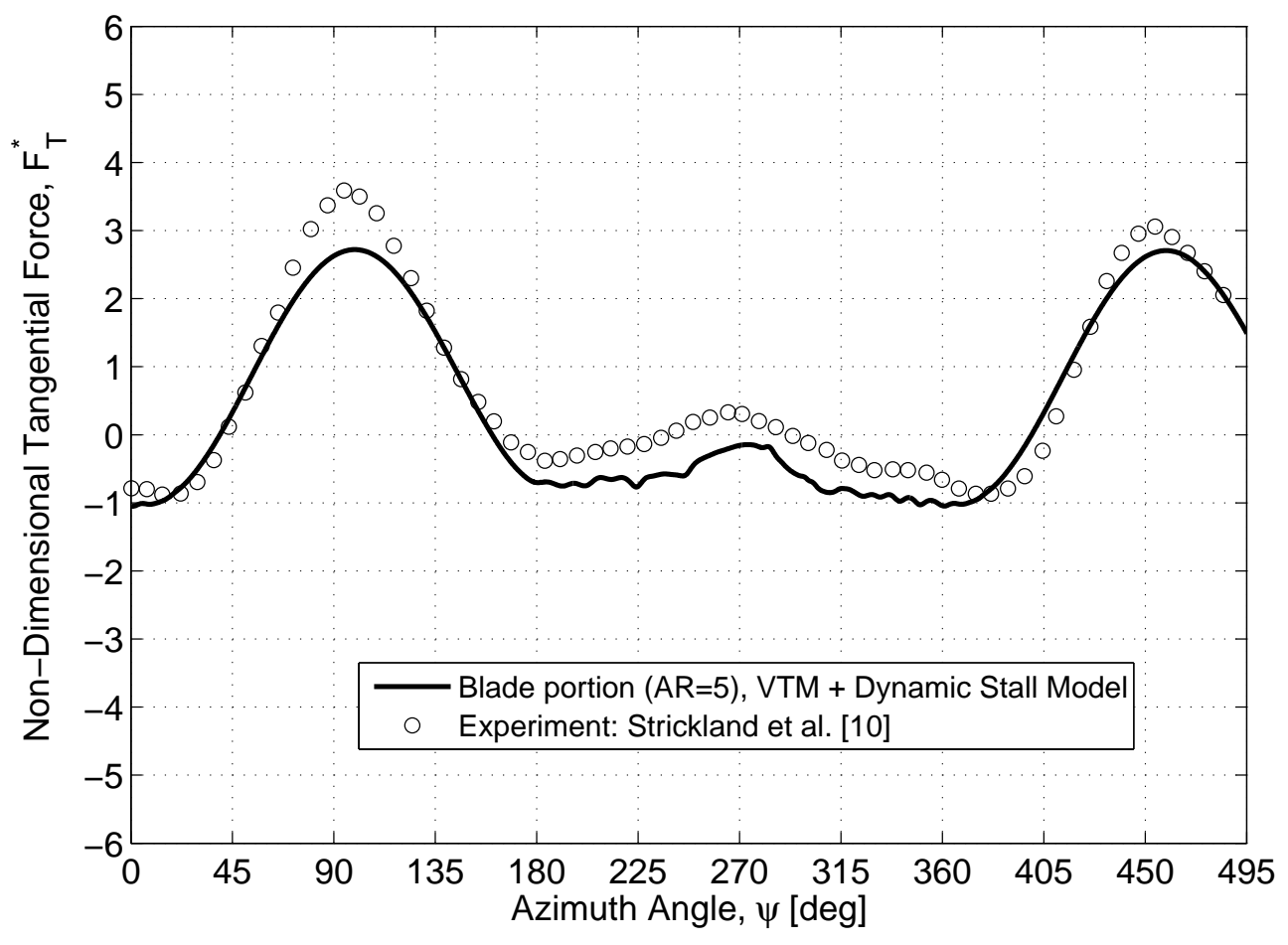

(b) Tangential force

Figure 14: Non-dimensional normal force (a) and non-dimensional tangential force (b). Comparsion between experimental measurements and VTM-simulations that include a dynamic stall model. 


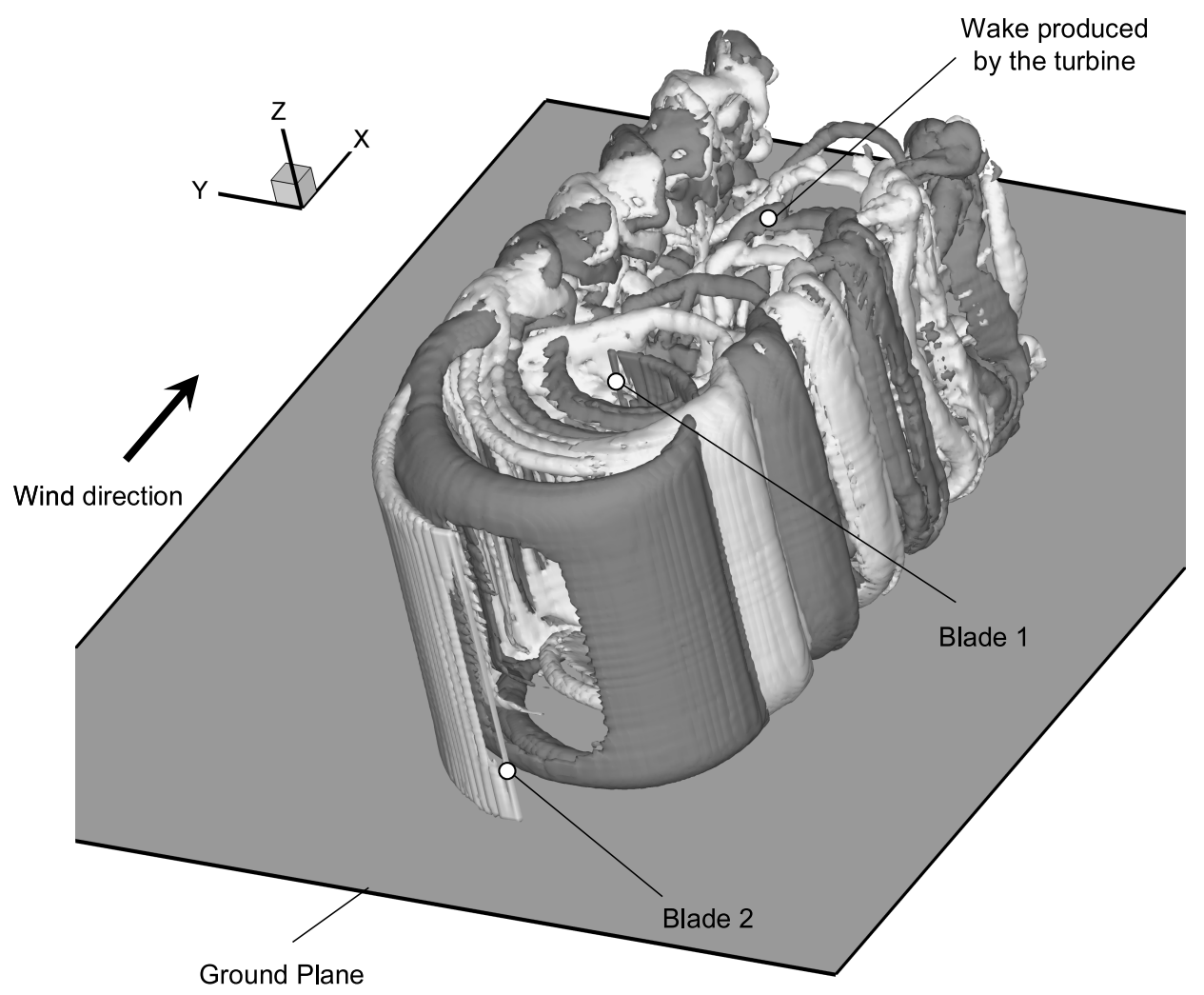

(a) Propagating wake produced by the turbine.

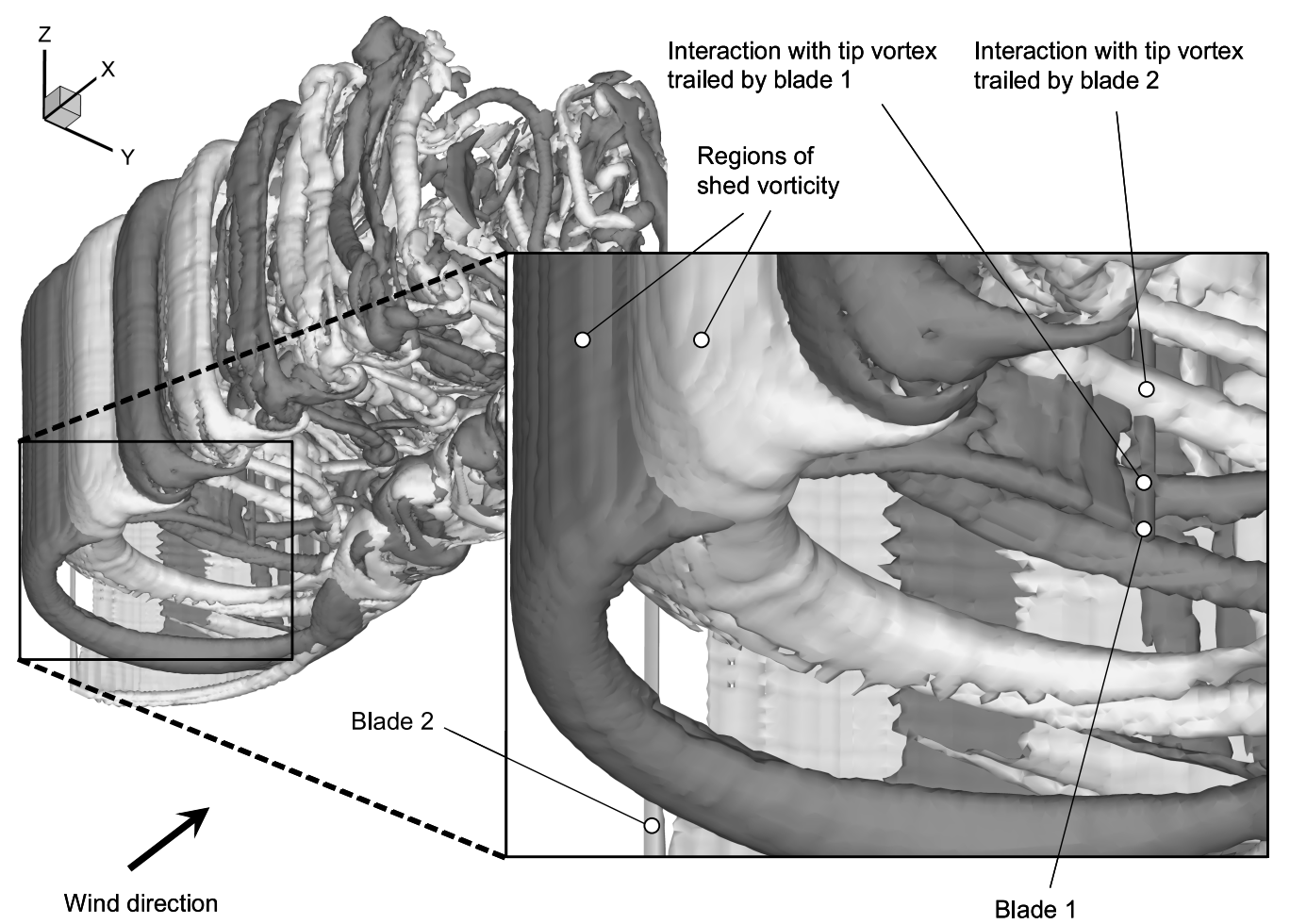

(b) Interaction between blade 1 and its own trailed vortex and the tip vortex trailed by blade 2.

Figure 15: Flow field visualisation by isosurface of vorticity with colouring allocated to the vorticity developed by blade 1 and blade 2. Blade 1 is located at azimuth $\psi=270^{\circ}$. 